\title{
In vivo evidence of microstructural hypo- connectivity of brain white matter in 22q11.2 deletion syndrome
}

Erika Raven ( $\sim$ Erika.Raven@nyulangone.org )

New York University

Jelle Veraart

New York University https://orcid.org/0000-0003-0781-0420

Rogier Kievit

MRC CBSU https://orcid.org/0000-0003-0700-4568

\section{Sila Genc}

Cardiff University

Isobel Ward

Cardiff University

Adam Cunningham

Cardiff University https://orcid.org/0000-0002-9791-7813

Joanne Doherty

Cardiff University

Marianne van den Bree

Cardiff University

Derek Jones

Cardiff University

\section{Article}

Keywords: 22q11.2 Deletion Syndrome, 22q11.2DS, white matter microstructure, microstructural connectivity

Posted Date: October 28th, 2020

DOI: https://doi.org/10.21203/rs.3.rs-80221/v1

License: (c) (1) This work is licensed under a Creative Commons Attribution 4.0 International License.

Read Full License 


\section{In vivo evidence of microstructural hypo-connectivity of brain white matter in 22q11.2 deletion syndrome}

Erika P. Raven ${ }^{1,2 *}$, Jelle Veraart ${ }^{2}$, Rogier A. Kievit ${ }^{3,4}$, Sila Genc ${ }^{1}$, Isobel Ward ${ }^{1}$, Adam Cunningham $^{5}$, Joanne Doherty ${ }^{5}$, Marianne B.M. van den Bree ${ }^{5 \dagger}$ Derek K. Jones ${ }^{1 \dagger}$

${ }^{1}$ Cardiff University Brain Research Imaging Centre (CUBRIC), School of Psychology, Cardiff University, Cardiff, UK

${ }^{2}$ Bernard and Irene Schwartz Center for Biomedical Imaging, Department of Radiology, New York University Grossman School of Medicine, New York, NY, USA

${ }^{3}$ Medical Research Council Cognition and Brain Sciences Unit, University of Cambridge, Cambridge, $U K$

${ }^{4}$ Cognitive Neuroscience Department, Donders Institute for Brain, Cognition and Behavior, Radboud University Medical Center

${ }^{5}$ Medical Research Council Centre for Neuropsychiatric Genetics and Genomics, Division of Psychological Medicine and Clinical Neurosciences, Cardiff University, Cardiff, UK

${ }^{6}$ Mary MacKillop Institute for Health Research, Australian Catholic University, Melbourne, Victoria, Australia

\footnotetext{
*Corresponding author: erika.raven@nyulangone.org
}

$\dagger$ These authors contributed equally to the work 
22q11.2 Deletion Syndrome, or 22q11.2DS, is a genetic syndrome associated with high rates of schizophrenia, autism, and attention deficit hyperactivity disorder, in addition to widespread structural and functional abnormalities throughout the brain. Experimental animal models have identified neuronal connectivity deficits, e.g., decreased axonal length and complexity of axonal branching, as a primary mechanism underlying atypical brain development in 22q11.2DS. However, it is still unclear whether deficits in axonal morphology can also be observed in people with 22q11.2DS. Here, we provide an unparalleled in vivo characterisation of white matter microstructure in both typically-developing children and children with 22q11.2DS using a dedicated magnetic resonance imaging scanner which is sensitive to axonal morphology. By extracting a rich array of diffusion metrics, we present microstructural profiles of typical and atypical white matter development, and provide new evidence of connectivity differences between typically-developing and 22q11.2DS children. A recent, largescale consortium study identified higher diffusion anisotropy and reduced overall mobility of water as hallmark microstructural alterations of white matter in 22q11.2DS, in particular for commissural fibers. We observed similar findings across all white matter tracts in this study, in addition to identifying deficits in axonal morphology. This, in combination with reduced tract volume measurements, supports the hypothesis that microstructural connectivity in 22q11.2DS is mediated by densely packed axons with disproportionately small diameters. Our findings provide insight into the in vivo mechanistic features of 22q11.2DS, and promote further investigation of shared features in neurodevelopmental and psychiatric disorders. 


\section{Introduction}

Neurodevelopmental disorders, including autism spectrum disorder (ASD), schizophrenia and attention deficit hyperactivity disorder (ADHD) have considerable overlap in their etiologies and clinical presentations, motivating the growing consensus that shared pathophysiological mechanisms are responsible for observed behavioral and cognitive deficits ${ }^{1-3}$. However, it has been a challenge to characterize potential mechanisms underlying these disorders due to their genetic and environmental heterogeneity. Therefore, by first studying individuals at elevated risk for neurodevelopmental or psychiatric disorders due to known genetic lesions, e.g., copy number variants (CNV), we can establish a critical link towards characterizing mechanisms involved in atypical development.

$22 \mathrm{q} 11.2$ deletion syndrome, or 22q11.2DS, is caused by a $1.5-3 \mathrm{Mb}$ deletion on chromosome 22. This CNV occurs in at least 1 in 4000 live births, making it the most prevalent chromosomal microdeletion syndrome ${ }^{4}$. Beyond recurrent physical attributes, including cardiac malformations, facial dysmorphology, immune deficiency and seizures/epilepsy, children with 22q11.2DS have a significantly enhanced risk of neurodevelopmental disorders, including learning disability, ASD, ADHD and developmental coordination disorder, and psychiatric disorders, including anxiety and depression ${ }^{5-8}$. In addition, the $22 \mathrm{q} 11.2$ deletion is recognized as the strongest known molecular risk factor for the development of schizophrenia (ranging from $23-43 \%$ of affected individuals) ${ }^{5,9}$.

Experimental animal models have demonstrated neuronal connectivity deficits are a highly penetrant feature of $22 \mathrm{q} 11.2 \mathrm{DS}{ }^{10}$. This may be linked to disturbed energy production, resulting 
in decreased proliferation and disrupted morphology of axons, dendrites, and synapses ${ }^{11,12}$. In turn, this leads to reduced connectivity of long-range white matter tracts and impaired synchrony of neural activity ${ }^{13}$. The development of white matter requires the complex coordination of neuronal and glial cell types to fine-tune the timing and synchrony of action potentials throughout the brain ${ }^{14-16}$. This includes subtle variations in axonal morphometry and packing ${ }^{17}$, myelination ${ }^{18}$, synaptic densities and neurochemical mediators. Thus, a better understanding of the neurobiological underpinnings of atypical brain development and connectivity in white matter requires continued investigation into in vivo scanning techniques sensitive to tissue microstructure.

Magnetic resonance imaging (MRI) has been used extensively in neurodevelopmental and clinical studies ${ }^{19-21}$, due to its sensitivity to subtle alterations in brain morphology and microstructure. Diffusion-weighted MRI (dMRI) is a particularly suitable technique as the signal reflects diffusion of water molecules on the micrometer length scale ${ }^{22,23}$. Thus, water acts as an in vivo probe, sensing obstacles constructed by cellular membranes and local fiber architecture ${ }^{23-26}$. It is the ubiquity of water, however, that typically challenges the biophysical interpretation of the dMRI signal collected with commonly available scanning equipment.

Conventional dMRI metrics have been shown to be very sensitive to a vast array of biophysical processes in tissue microstructure, but specificity is distinctly lacking. This limitation can now be overcome by exploiting unusually strong diffusion-weightings that have only recently become available through the development of ultra-strong MRI gradients for human neuroimaging ${ }^{27,28}$. At such strong diffusion weightings (i.e., high $b$-values), the dMRI signal is dominated by contribu- 
tions from water within cellular compartments such as axonal or glial processes. Moreover, there has been a proliferation of biophysical models of diffusion in tissue that hold the promise of quantifying important cellular features such as axonal diameters, densities and orientational dispersion in vivo ${ }^{29}$ which benefit hugely from the implementation of ultra-strong gradients.

To explore axonal morphology in depth, it is necessary to move beyond current state-of-theart MRI techniques (e.g., diffusion tensor MRI, or DTI) to achieve enhanced cellular specificity in developmental populations. Here, we present an in-depth characterization of white matter microstructure in both typically-developing and 22q11.2DS participants using ultra-strong gradients and an extensive multi-shell dMRI acquisition, including $b$-values up to $6000 \mathrm{~s} / \mathrm{mm}^{2}$. By so doing, we conducted a multi-parametric analysis to gain novel insights into the microstructural properties underlying disrupted axonal morphology, to better describe previous observations of white matter hypo-connectivity in 22q11.2DS

The findings can provide insight into 1) disentangling microstructural connectivity differences in brain white matter in 22q11.2DS versus typical developing participants, and 2) establishing the sensitivity and specificity of in vivo dMRI to underlying biophysical mechanisms in hypo-connectivity of white matter.

\section{Methods and Materials}

Subjects This study included 92 typically-developing (TD) children (age = 8-18 years, F=49) and 6 children with 22q11.2DS ( $\mathrm{F}=6$ ) (see Table 1 for full demographic information). From the TD 
group, a subset of children were selected based on age and sex ( $\mathrm{n}=14$, age- and sex- matched group, ASM) for group comparison to children with 22q11.2DS. The Cardiff University School of Psychology Ethics Committee approved data collection procedures for the TD group, and each participant and/or their parent or legal guardian provided written informed consent. TD participants were recruited from the Cardiff area, and screened to exclude major neurological disorders, such as epilepsy. Participants with 22q11.2DS were recruited from the Experiences of CHildren with cOpy (ECHO) number variants study at Cardiff University (https://bit.ly/Cardiff-Echo-Study) ${ }^{30-32}$. ECHO study protocols were approved by NHS Wales Research Ethics Committee and brain imaging procedures were approved by the Cardiff University School of Medicine Ethics Committee. As part of the ECHO study, 22q11.2DS participants underwent extensive psychiatric, developmental and cognitive assessment including IQ assessment using the Weschler Abbreviated Scale Intelligence (WASI-II, ${ }^{33}$ ). The ASM control group also performed an abbreviated 2-subtest WASI to establish a normative reference point in IQ scores. 22Q11.2DS also provided information about social communication difficulties indicative of ASD from the Social Communication Questionnaire $\left(\mathrm{SCQ},{ }^{34}\right)$, and psychiatric interviews were taken using the Child and Adolescent Psychiatric Assessment $\left(\mathrm{CAPA},{ }^{35}\right)$. Seizure and medication history were obtained from parental questionnaires ${ }^{36}$. One child had a history of febrile seizures, but no other seizure history or psychiatric/epilepsy medications were reported. All participants underwent the same pre-scan procedure, which included an in-person screening for MRI safety and a practice MRI scan in a mock MRI scanner 37. 
Image acquisition and preprocessing All imaging data were acquired on a 3T Connectom scanner (Siemens Healthcare, Erlangen, Germany) with $300 \mathrm{mT} / \mathrm{m}$ gradients and a 32-channel radiofrequency coil (Nova Medical, Wilmington, MA, USA) at the Cardiff University Brain Research Imaging Centre (CUBRIC).

T1-weighted anatomical images were acquired using a 3D Magnetization Prepared Rapid Gradient Echo (MP-RAGE) sequence with the following parameters: echo time of 2 ms, repetition time of $2300 \mathrm{~ms}$, time to inversion of $857 \mathrm{~ms}$, and flip angle of $9^{\circ}$. The field of view was $256 \times 256$, the imaging matrix $256 \times 256$, with 192 slices of $1 \mathrm{~mm}$ slice thickness. The total scan time was 5 minutes and 32 seconds.

dMRI data were acquired using a multi-shell diffusion-weighted EPI sequence. Images covered the whole brain with field of view $220 \times 220 \mathrm{~mm}$, imaging matrix $110 \times 110$, and 66 slices of $2 \mathrm{~mm}$ slice thickness. dMRI were acquired using the Stejskal-Tanner sequence with an anteriorto-posterior phase-encoding direction, with an echo time of $59 \mathrm{~ms}$, repetition time of $3000 \mathrm{~ms}$, and with diffusion-weighting gradient separation $\Delta=23.3 \mathrm{~ms}$ and duration $\delta=7 \mathrm{~ms}$. Data were acquired at five different $b$-values: $b=500,1200,2400,4000$, and $6000 \mathrm{~s} / \mathrm{mm}^{2}$; with 30 and 60 non-collinear directions used for $b \leq 1200 \mathrm{~s} / \mathrm{mm}^{2}$ and $b \geq 2400 \mathrm{~s} / \mathrm{mm}^{2}$, respectively ${ }^{38}$. In addition, 14 non-diffusion weighted images were distributed uniformly throughout the protocol. One additional volume was acquired with reversed phase encoding for the purpose of EPI distortion correction. The total dMRI scan time was 16 minutes and 14 seconds.

The following preprocessing steps were used to reduce thermal noise and image artifacts: 
image denoising ${ }^{39}$, correction for signal drift ${ }^{40}$, motion, eddy current, and suspecptibility-induced distortion correction ${ }^{41}$, gradient non-linearities, Gibbs ringing ${ }^{42}$, and outlier rejection ${ }^{43}$. The preprocessing pipeline was implemented in MATLAB (The MathWorks, Natwick, MI, USA), and depended on open source software packages from MRtrix ${ }^{44}$ and FSL ${ }^{45}$.

Quantitative diffusion metrics There has been little consensus as to which dMRI metric provides the most sensitive or specific characterisation of the white matter's biophysical properties. In this work, we use an inclusive analysis approach rather than focus on a single analysis strategy, examining both mathematical representations and biophysical models of the dMRI signal ${ }^{29}$. The former have been shown to be sensitive to tissue microstructure, but intrinsically suffer from poor specificity to microstructural variations. In contrast, biophysical models that aim to bypass this limitation by assigning different components of the signal to distinct tissue compartments/properties, e.g. intra- and extra-cellular spaces ${ }^{46,47}$, may improve specificity but often lack sensitivity. In our study, the richness of the diffusion-weighted data acquisition enabled us to adopt a minimally constrained biophysical model, thereby minimizing previously-reported biases arising from unvalidated or contested assumptions ${ }^{48}$. The representations and biophysical model parameters included in our analyses are outlined below.

- Diffusion tensor and kurtosis imaging: DTI ${ }^{49}$ and diffusion kurtosis imaging (DKI) ${ }^{50}$ have been used extensively in studies of white matter microstructure in typical and atypical development (see review, ${ }^{20}$ ). DTI metrics include fractional anisotropy (FA), mean diffusivity $(\bar{D})$, radial diffusivity $\left(D_{\perp}\right)$ and axial diffusivity $\left(D_{\|}\right)$. DKI metrics include mean kurtosis 
$(\bar{K})$, radial kurtosis $\left(K_{\perp}\right)$ and axial kurtosis $\left(K_{\|}\right)$, and are shown to be intrinsically more sensitive to compartmental microstructural attributes than DTI parameters (see review, ${ }^{51}$ ). The diffusion and kurtosis tensors were estimated from the data with $b \leq 2400 \mathrm{~s} / \mathrm{mm}^{2}{ }^{52}$. To improve the robustness of these parameters, prior to fitting the kurtosis tensor, the data were first smoothed with an anisotropic Gaussian filter ${ }^{53}$ that was aligned with the underlying fiber architecture.

- Spherical moments: Without imposing any biophysical assumptions or prior knowledge, we compute the spherical mean and spherical variance of the diffusion-weighted MRI signal for each distinct diffusion weighting, $b=500,1200,2400,4000$, and $6000 \mathrm{~s} / \mathrm{mm}^{2}$ using the power of the $0^{\text {th }}$ and $2^{\text {nd }}$ order spherical harmonic coefficients ${ }^{54}$. At high $b$-values, e.g. $b=6000 \mathrm{~s} / \mathrm{mm}^{2}$, the spherical mean $\stackrel{\circ}{S}_{\mu}(b)$ and spherical variance $\stackrel{\circ}{S}_{\sigma}(b)$ relate strongly with specific information about the intra-cellular microstructure, including the axon diameter and the intracellular signal fraction and diffusivity (See Suppl. Section Sensitivity to the axon morphology) ${ }^{55}$. Unlike $\stackrel{\circ}{S}_{\mu}(b), \stackrel{\circ}{S}_{\sigma}(b)$ also encodes the orientational dispersion ${ }^{48}$.

- Biophysical Models: Here, we adopt the Biophysical Standard Model (BSM) of white matter to extract more specific biophysical features. The model describes restricted diffusion within dispersed neuronal and/or glial processes, embedded in an effective extra-cellular matrix ${ }^{48}$. The model is parameterized by intracellular signal fraction $(f)$, parallel intracellular diffusivity $\left(D_{c}\right)$, parallel and perpendicular extracellular diffusivity $\left(D_{e}^{\|}, D_{e}^{\perp}\right)$, and orientational alignment $(\kappa)$. Note $0 \leq \kappa \leq 1$ with $\kappa=0$ for fully isotropic diffusion cf. $p_{2}$ in Novikov et al. ${ }^{48}$. 
The precision of the estimation of the individual metrics is evaluated in a simulation experiment; see Suppl. Section Precision of diffusion metrics.

Segmentation Digital brain extraction was performed using the FSL Brain Extraction Tool (BET) $)^{45}$ followed by the FSL segmentation tool (FAST) ${ }^{56}$ in which three tissue types, i.e., CSF, WM, and GM were segmented. Moreover, automated tract segmentation software was used to extract eleven white matter fiber tracts using the $b=6000 \mathrm{~s} / \mathrm{mm}^{2}$ shell for each participant ${ }^{57}$. Tract selection was referenced from previous developmental neuroimaging studies using dMRI ${ }^{20}$. These included association tracts: arcuate fasciculus (AF), cingulum (CG), inferior fronto-occipital fasciculus (IFO), inferior longitudinal fasciculus (ILF), superior longitudinal fasciculus (SLF), and uncinate fasciculus (UF); commissural tracts: genu (GCC), body (BCC), and splenium (SCC) of the corpus callosum; and projection tracts: corticospinal tract (CST), optic radiation (OR). All tract reconstructions were bilateral except for the inter-hemispheric projections of the corpus callosum; see Suppl. Fig. S1

Tract volume quantification The volume of each segmented fiber pathway was estimated by creating a tract density map using the associated streamlines ${ }^{58}$, then counting the total number of voxels in which streamlines were observed. Nominal tract volumes were normalized for total brain volumes to compensate for brain size differences. Since each individual streamline is strongly susceptible to thermal noise, the estimation of volume quantification might be less accurate for fiber pathways with high surface-to-volume ratio. For our cohort, the minimally detectable effect size is 10 to $20 \%$ (cf. Suppl. Section Tract Volume Quantification). Therefore, the sensitivity of the 
technique is intrinsically limited to relatively large changes in typical and/or atypical development.

Along tract profiling The estimation of the BSM parameters on a voxel level is challenging because of the poor precision and degeneracy in the model at such a low signal-to-noise ratio (SNR), i.e., multiple solutions are possible for one signal ${ }^{59}$. However, by adopting the novel approach of averaging the (rotationally-invariant) spherical moments within tract segments prior to model fitting, we were able to boost the SNR of our data and thus the reliability of BSM parameter estimates, thereby fostering more accurate and precise biophysical modeling. Note, the curvature of tracts does not bias the model parameters because they are derived from the rotationally invariant spherical moments of the signal.

Specifically, we projected the rotationally-invariant spherical moments of each individual tract onto twenty equidistantly-spaced nodes of the associated centerline. This in-house strategy was implemented in MATLAB, inspired by tractometry methods such as the AFQ toolbox and others ${ }^{60,61}$. Each centerline was computed by fitting a three-dimensional spline through equidistantlyspaced sample points of 10,000 streamlines that constituted an individual bundle ${ }^{60}$. The intrinsic challenges of fiber tracking, i.e., spurious connections, might propagate in this analysis ${ }^{62}$. Therefore, quality control was performed using visual inspection of centerline geometry and positioning. From this, we identified the body of the corpus callosum, or BCC, to be poorly robust due to streamlines occurring in spurious directions. The BCC was removed from subsequent comparisons of 22q11.2DS participants. In addition, along-tract metric profiles for all subjects were aligned to account for varying tract lengths and head sizes using a linear translation and rescaling 
of the nodes.

Statistical Analysis Statistical analyses were conducted using R v3.4.1 (Single Candle) ${ }^{63}$ and MATLAB. We considered each of the metrics outlined above as each varies in terms of its accuracy, precision and specificity. However, redundancy across parameters is intrinsic to diffusion MRI; thus, a principal component analysis (PCA) was used to identify latent structure in total white matter estimates of the TD cohort. While PCA can account for multi-collinearity in predictor variables ${ }^{64}$, we excluded estimates of the spherical mean and spherical variance derived at intermediate $b$-values $\left(1200,2400,4000 \mathrm{~s} / \mathrm{mm}^{2}\right)$ to preemptively minimize redundancy in the analysis ${ }^{65}$. The data sampling adequacy was assessed using a Kaiser-Meyer-Olkin (KMO) test and the Bartlett's test of sphericity. Parallel analysis was used to select the optimal number of principal components. We used an oblique matrix rotation to allow underlying biophysical components to correlate, as this is likely most biologically plausible. The loadings per principal component were used to compute a composite total, or PCA score.

The associations between age and PCA scores/individual metrics within selected tracts were assessed using the the goodness-of-fit for both linear (age) and quadratic (age ${ }^{2}$ ) models as reported in the literature for developmental cohorts ${ }^{20}$. Using the corrected Akaike information criterion (AIC) to compare linear versus quadratic models, we found no statistical evidence to motivate the more complex (i.e., quadratic) model for this age range. Therefore, all age-associations for PCA scores/individual metrics in total white matter and tracts were assessed using a simple linear regression model for age. Sex was not included as a predictor in these analyses as the 22q11.2DS 
and ASM groups are all female. Model statistics for each comparison with age are reported as the $r^{2}$ and unstandardized $\beta$ coefficient. The statistical significance level $(p \leq 0.035)$ is determined using the Benjamini-Hochberg procedure to control the false discovery rate (FDR) in this study ${ }^{66}$. Predicted metric scores were computed using the regression equation for youngest (8 year old) and oldest (18 year old) participants. The difference in predicted metric scores was used to illustrate age-effects in the TD group.

Standardized effect size was computed between age- and sex-matched controls (ASM) and 22q11.2DS participants. Here, we report Hedge's $g$, which corrects for any bias that might be present due to small sample sizes ${ }^{67}$. The statistical significance level is $p \leq 0.011$ after FDR correction. The $t$-statistic and degrees of freedom $d f(N-1)$ are reported for independent group comparisons.

\section{Results}

Biophysical principal components of white matter microstructure Fig. 1 shows the results of the PCA performed to characterize biophysical constituents of white matter microstructure during development. Parallel analysis in total white matter suggested that three components (dubbed here 'Biophysical Principal Components' or BPCs) derived from eleven dMRI metrics was adequate to explain the data, together explaining $91 \%$ of the total variance in white matter. Parallel analysis was also applied to individual tracts, again confirming three components with the same groups of variables. Data sampling adequacy was verified by the KMO-statistic, $\mathrm{KMO}=0.68$, and Bartlett's 
test of sphericity, $x(55)=2005, p<0.05$. Table 2 shows the standardized component loadings of each metric after oblique rotation.

Inspection of the loading matrix showed that each of the three BPC's could be described as capturing a specific biophysical dimension of microstructural features in white matter, as indicated by high component loadings for groups of variables $(>0.5)$ (Fig. 2). Here, we introduce labels to aid the interpretation of these features: intracellular signal (BPC1), extracellular mobility (BPC2), and tissue complexity (BPC3). The motivation of this BPC labels is provided in Suppl. Section The biophysical interpretation of the BPC. The first component, BPC1, captured $31 \%$ of the variance in the data, and was characterized most strongly by high loadings FA, $\stackrel{\circ}{S}_{\mu}(b=6000), \stackrel{\circ}{S}_{\sigma}(b=500)$, and $\stackrel{\circ}{S}_{\sigma}(b=6000)$. The second component, BPC2, captured $33.1 \%$ of variance in the data, with high loadings for $\bar{D}, D_{\perp}$, and $D_{\|}$, and $\stackrel{\circ}{S}_{\mu}(b=500)$. And finally, the third component, BPC3, captured $27.7 \%$ variance in the data, with high loadings for $\bar{K}, K_{\perp}$, and $K_{\|}$. Regression component scores showed sensitivity to age for all BPCs; however, the strongest association with age was found to be tissue complexity (BPC $3 ; r^{2}=0.369, \beta=0.002, p<0.0001$ ). This was closely followed by intracellular volume (BPC1; $\left.r^{2}=0.325, \beta=0.003, p<0.0001\right)$, and then extracellular mobility (BPC2; $\left.r^{2}=0.222, \beta=-0.002, p<0.0001\right)$, and (Fig. 1A).

In the 22q11.2DS participants (average age: $13.1+/-1.0$ years), the values of BPC1 and BPC2 were aligned to those predicted for TD participants at 18 years of age, suggesting a more mature dMRI signature of development. Compared with ASM children, 22q112DS children had higher intracellular signal fraction $(\mathrm{BPC} 1 ; t(18)=4.884, p<0.001, g=2.282$ ), and lower 
extracellular mobility (BPC2; $t(18)=-2.186, p<0.05, g=1.021)$ (Fig. 1B). There were no significant differences in tissue complexity between 22q11.2DS and ASM participants.

Sensitivity and specificity of individual dMRI metrics To put the BPCs into context with existing diffusion literature, individual dMRI metrics were also explored in typical and atypical development.

\subsubsection{Typical Development}

After fitting the linear age-dependence for total white matter, metrics strongly associated with intracellular volume (BPC1), i.e., $\stackrel{\circ}{S}_{\mu}$ and $\stackrel{\circ}{S}_{\sigma}$ at high $b$-values, had the largest differences versus all other metrics in predicted values between ages 8 and 18 of $\sim 20 \%$. This large effect was reflected by strong associations with age $\left(\stackrel{\circ}{S}_{\mu}(b=6000): r^{2}=0.553, \beta=0.002, p<0.0001\right.$; and $\left.\stackrel{\circ}{S}_{\sigma}(b=6000): r^{2}=0.369, \beta=0.0002, p<0.0001\right)$ (Fig. 2). Significant age-associations were also found at the tract level for both metrics, as shown in Fig. 2C. In comparison, total white matter $\stackrel{\circ}{S}_{\sigma}$ at low $b$-value and FA were less sensitive to age effects $\left(\stackrel{\circ}{S}_{\sigma}(b=500): r^{2}=0.120\right.$, $\beta=0.0001, p=0.001$; and FA: $\left.r^{2}=0.165, \beta=0.002, p=0.0002\right)$ and had negligible effects at the individual tract level.

For extracellular mobility metrics $(\mathrm{BPC} 2), \bar{D}, D_{\perp}$, and $\stackrel{\circ}{S}_{\mu}(\mathrm{b}=500)$ had significant associations with age in total white matter $\left(\bar{D}: r^{2}=0.216, \beta=-0.004, p<0.0001 ; D_{\perp}: r^{2}=0.294\right.$, $\left.\beta=-0.005, p<0.0001 ; \stackrel{\circ}{S}_{\mu}(b=500): r^{2}=0.248, \beta=0.001, p<0.0001\right)$. These associations 
were also present for most tracts. $D_{\|}$did not have any notable associations with age for total white matter or individual tracts.

Tissue complexity metrics (BPC3) had high sensitivity to age for total white matter and most individual tracts. In total white matter, $K_{\|}$had the strongest association $\left(K_{\|}: r^{2}=0.55, \beta=0.005\right.$, $p<0.0001)$, followed by $\bar{K}\left(r^{2}=0.28, \beta=0.007, p<0.0001\right)$ and $K_{\perp}\left(r^{2}=0.16, \beta=0.008\right.$, $p=0.0002)$

Biophysical modelling was used to complement the sensitivity of BPCs and their composite metrics with more specific measures ${ }^{48}$. From the BSM, the intracellular signal fraction $(f)$ showed the greatest sensitivity to age in total white matter $\left(r^{2}=0.299, \beta=0.005, p<0.0001\right)$, being $\sim 9 \%$ higher over the age range. These effects were significant for most tracts with percent differences ranging from $\sim 3$ to $16 \%$. The $D_{e}^{\perp}$ and $\kappa$ were lower with age in total white matter $\left(D_{e}^{\perp}: r^{2}=0.094, \beta=-0.005, p=0.005 ; \kappa: r^{2}=0.019, \beta=-0.0007, p=0.11\right)$, although $\kappa$ did not reach significance here. $D_{c}$ and $D_{e}^{\|}$were not sensitive to age effects in this cohort. While biophysical modelling holds the promise of more specific interpretation, it is challenged by lower precision in BSM parameter estimates. In Suppl. Fig. S2, the precision of metrics and BSM model parameters is shown, highlighting this discrepancy, e.g., $D_{c}$ and $D_{e}^{\|}$. Therefore, the combination of both sensitive and specific dMRI metrics allows for more in-depth phenotyping of white matter microstructure. 


\subsubsection{2q11.2DS}

As shown in Fig. 4, compared to ASM controls, participants with 22q11.2DS exhibited marked differences in microstructure parameters. For intracellular signal, all metrics, not just those derived from high $b$-value data, were sensitive to atypical development with differences ranging from $\sim$ $8-11 \%$ in total white matter. While the 22q11.2DS group was small, possibly leading to inflated effect sizes, these differences were consistently found to be very large ( $g=1.9$ to 2.4$)$. Individual tracts reflected a global trend, with only UF, OR, and SCC having smaller, albeit significant effects in these metrics. An FDR correction did not alter the significance of the effects. For extracellular mobilities, $\bar{D}$ and $D_{\perp}$ were most dominant, being $5-7 \%$ lower in total white matter for 22q11.2DS participants. Effect sizes in total white matter were large (MD: $g=1.0 ; D_{\perp}: g=1.4$ ); which was also observed for individual tracts. For $D_{\|}$and $\stackrel{\circ}{S}_{\mu}$ at low- $b$, differences ranged from $\sim 1-2 \%$; however, effect sizes were large in total white matter $\left(D_{\|}: g=0.5 ; \stackrel{\circ}{S}_{\mu}(b=500) g=1.2\right)$ and individual tracts. Tissue complexity metrics were the least sensitive metric class to atypical development.

Of BSM modelling parameters, 22q11.2DS participants had $\sim 6 \%$ higher $f, \sim 10 \%$ lower $D_{e}^{\perp}$, and $\sim 2.5 \%$ lower $\kappa$ versus the ASM control group. Similar to the TD cohort, $D_{c}$ and $D_{e}^{\|}$were not sensitive to differences, possibly reflecting the poor precision of these metrics (Suppl.Fig S2). At the individual tract level, $f$ and $D_{e}$ also had the strongest effects in detecting group differences. 
Whole brain and tract volumes Whole brain and individual tract volumes were computed for both TD and 22q11.2DS groups, see Fig. 3. For the TD group, there were significant age effects for lower GM volume and higher CSF volume with age (GM: $r^{2}=0.536, \beta=-0.007, p<0.0001$;

CSF: $r^{2}=0.579, \beta=0.007, p<0.0001$ ), aligning with previous developmental literature ${ }^{68,69}$. There were no significant age effects for total brain volume or WM volume which is not consistent with other findings. In addition, the WM volume analysis per tract was not sensitive to age effects.

Compared to ASM, 22q11.2DS children had significantly higher CSF volume $(t(18)=$ 3.305, $p=0.004, g=1.544$ ), but no difference in whole brain, GM, or WM volumes (Fig. 3A, B). In contrast, as shown in Fig. 3C, there were significant reductions in most individual tract volumes for 22q11.2DS. Differences in tract volumes were large, ranging from $11-31 \%$, and were present irrespective of tract type or location. Fig. 3D highlights the global pattern of these differences across the brain.

\section{Discussion}

The results of this study support the hypothesis that disruptions in axonal morphology contribute to microstructural hypo-connectivity of white matter in children with 22Q11.2DS. This finding has been demonstrated in preclinical models post-mortem, but is observed here in humans for the first time in vivo. We achieved direct sensitivity to axonal morphology by using a combination of high- $b$ value diffusion MR and advanced biophysical modeling. The specificity of this approach is highlighted by 1) differences in 22Q11.2DS participants versus ASM controls, and 2) age-effects 
in TD children.

\subsubsection{In-depth microstructural phenotyping of white matter in 22q11.2DS}

The dMRI signal reflects a diverse array of microstructural attributes, including axonal density and packing, intra-voxel fiber orientational dispersion, membrane permeability, and myelination. Therefore, it is an inherent challenge to extract inferences on which specific process, or combination of processes, is most likely contributing to dMRI metrics. The utility of PCA and factor analysis techniques for grouping MRI measures in biophysically relevant ways has been demonstrated

previously ${ }^{70-73}$. Here, this approach allowed us to probe the sensitivity of dMRI to differences in 22q11.2DS while characterizing three distinct sources, or BPCs, representative of the underlying microstructure. Accepting the risk of oversimplification, we coined these BPCs as "intracellular signal", "extracellular mobility", and "tissue complexity".

By comparing the 22q11.DS to age- and sex-matched matched (ASM) controls, projecting the differences onto trends of typical development in adolescence, we conclude that children with 22q11.2DS had dMRI profiles most similar to older children, Fig. 1. This holds for intracellular signal (BPC1) and extracellular mobility (BPC2), in addition to their composite metrics for both total white matter and individual tracts, Fig. 4. A similar observation was recently made for conventional DTI metrics by a large, multi-site study on 22q11.2DS (ENIGMA-22q11DS working group $^{74}$ ). While DTI metrics reflect many contributions from the tissue microstructure, the aforementioned study reported marked differences in white matter regions of interest versus age- and 
sex-matched controls for participants aged 5 to 50 years. Notably, the direction and magnitude of effect sizes in FA (higher) and diffusivity metrics, $\bar{D}, D_{\perp}$, and $D_{\|}$(lower), were comparable to our observations, as shown in Fig. 4. The main exceptions were in three association regions the SLF, external capsule, and fornix where the authors observed lower FA. However, patterns of reduced diffusivity remained consistent for most regions. White matter development is heterochronistic, meaning different regions develop at different times and rates. In this way, association tracts have the most protracted development, continuing into the third decade of life and beyond ${ }^{16,18,75}$. It is possible that competing maturational processes such as ongoing myelination may confound inferences over a broad age range, leading to the observed differences.

In addition to the diffusion metrics quantifying microstructure, we observed significant morphological differences in white matter bundles. Specifically, we identified lower white matter volume in most pathways for 22q11.2DS, excluding the body and splenium of the CC and the UF. Such widespread decreases in white matter volumes are in agreement with previous studies of $22 q 11.2 \mathrm{DS}{ }^{21}$. We did not observe significant age-related differences in WM volume across the age-span studied here in the typically developing cohort. However, the reduction in GM volume and increase in CSF volume is consistent with the literature ${ }^{69}$. In what follows, we will argue that the combination of changes in dMRI metrics that were sensitized to axonal morphology at the micro-scale, and lower white matter volume in the 22q11.2DS participants at the macro-scale, provides evidence in support of the hypo-connectivity hypothesis in 22q11.2DS and a lower number of large axons in particular. 


\subsubsection{In support of hypo-connectivity}

To examine the biological plausibility of our findings, we can look at converging evidence from mouse models of 22Q11.2DS. For instance, Fernandez et al characterized under-connectivity as limited axon and dendrite growth, and disrupted mitochondria and synaptic integrity ${ }^{11}$. These effects were specific to neurons in layer $2 / 3$ of the cortex, which primarily support long-distance cortico-cortico connections. In a separate mouse model used to report on genetic susceptibility of schizophrenia, altered axonal growth, branching, and disrupted connectivity were linked to behavioral deficits ${ }^{12,13}$. In these studies, both short and long range connectivity was impacted. The size of terminal axonal branching correlates with the caliber of axons - therefore, large diameter axons are capable of high rates of information transfer at these junctions ${ }^{76-78}$. Axonal growth and branching is an energetically demanding process. Therefore, in 22q11.2DS, where mitochondrial dysfunction has been linked to atypical axonal morphology ${ }^{11}$, there may be a critical susceptibility for this subset of neurons. To date, we are not aware of any studies measuring axon diameter distributions in 22q11.2DS; however, studies of other neurodevelopmental disorders, such as ASD and Angelman Syndrome, have reported a lower number of large axons in comparison to typical devel-

opment ${ }^{79-81}$. Therefore, we hypothesize that the lower white matter tract volumes in 22q11.2DS are representative of densely packed axons with disproportionately small diameters. 


\subsubsection{Sensitivity to axonal morphology}

A unique feature of our study is the sensitivity to axonal morphology at $b=6000 \mathrm{~s} / \mathrm{mm}^{2}$ achieved by exploiting the ultra-strong gradients, i.e., $300 \mathrm{mT} / \mathrm{m}$, of the Connectom scanner. Our data did not allow for the direct quantification of the axon diameter (as discussed below in Considerations for scanning). However, various metrics are sensitive to the axon diameter and are, as such, directly sensitive to differences in the axon diameter distribution, with particular sensitivity to differences in the number of large axons ${ }^{82}$. The key parameters are the spherical mean and variance at the highest $b$-value. The significant increase in both parameters in the 22q11.2DS compared to ageand sex-matched controls is consistent with a reduction in mean axon diameter. Of relevance to our observation of higher FA in 22q11.2DS and in line with Villalón-Reina et al ${ }^{74}$, an association between higher FA (and smaller $D_{\perp}$ ) and smaller axons has previously been demonstrated ${ }^{83}$.

Moreover, there is an inverse relationship between axon diameter and axon density, i.e., larger diameter axons take up more space, due not only to larger size, but also from the space demands of neighboring glial cells such as myelinating oligodentrocytes and astrocytes ${ }^{84-86}$. When there are fewer large diameter axons, or their morphology is less complex due to fewer branch points, the space requirements go down - in agreement with the significant loss of tract-based white matter volumes seen in our study. As a consequence, the relative proportion of smaller diameter axons increases, leading to greater axonal density and the reduced $D_{e}^{\perp}$. Moreover, dMRI has a reduced (or even nullified) sensitivity to smaller axons as a result of which the dMRI signal is

less attenuated (see Suppl. Fig. S4). Both effects are consistent with higher values of $\stackrel{\circ}{S}_{\mu}$ and $f$ as 
seen in 22q11.2DS relative to ASM controls. Finally, higher orientational dispersion, (i.e., drop in orientational-concentration parameter $\kappa$ ), in combination with lower axial diffusivity, might reflect a more tortuous path of axons that has been associated with smaller axons ${ }^{87,88}$. This hypothesis was also put forward by the ENIGMA working group based on the lower axial diffusivities in their study ${ }^{74}$. However, a more direct measurement of tortuosity along segments of axons depends on $D_{c}$, a metric that was not estimated with sufficient precision using the Biophysical Standard Model to support this hypothesis.

\subsubsection{Typical development}

Although this work focuses on 22q11.2DS, our results also provide new insights into typical development. There is ongoing debate about the biophysical underpinnings of brain maturation: axonal growth versus myelination ${ }^{89}$. To this end, the extended multi-shell dMRI data, including the high $b$-values, complement previous observations of enhanced sensitivity to age effects (e.g., $b=3000$ )

90. Notably, from the high $b$-value data, $\stackrel{\circ}{S}_{\mu}$ and $\stackrel{\circ}{S}_{\sigma}$ are the most sensitive parameters amongst all evaluated metrics. Given that the signal from the extra-cellular space is effectively filtered out at these b-values, this result suggests that the dynamics of the neuronal and/or glial processes, rather than the extra-cellular signal, are dominating effects related to age-dependency in dMRI. As suggested by ${ }^{89}$, white matter maturation might encompass axonal growth or swelling due to neuronal activity ${ }^{91,92}$ or even hormonal fluctuations ${ }^{93,94}$. However, the increase in axon diameters exclusively would have resulted in a lower $\stackrel{\circ}{S}_{\mu}(b=6000)$ (see Suppl. Fig. S4). We observed the 
opposite; therefore, higher values of $\stackrel{\circ}{S}_{\mu}(b=6000)$ and $f$ are dominated by the greater density of neuronal and/or glial processes. In addition, we observed less alignment of these processes, $\kappa$. This particular combination supports the greater density of more orientationally-dispersed cellular processes, such as those arising from glial cells. Additionally, the omnidirectional increase in kurtosis and omnidirectional reduction in mobility in the extra-cellular space are both in agreement with a greater cellular density.

Based on these observations, we hypothesize that increased complexity in astrocytic processes may be contributing to the age-dependency of the diffusion-weighted signal in typically developing children. A detectable change in astrocytes might be expected because of their abundance in the white matter ${ }^{77}$, the size and complexity of their processes ${ }^{86}$, and their critical role in protracted white matter development ${ }^{95-97}$. While oligodendrocytes have been well characterized in development and are known to be fundamental for myelin formation and maintenance ${ }^{98-100}$, astrocytes also have many important roles, including providing trophic support of oligodendrocytes, formation of the blood brain barrier, synaptic pruning and neurotransmitter recycling ${ }^{101}$. Moreover, it has been demonstrated that astrocytes go through morphological and density changes during brain maturation ${ }^{95,102}$. Diffusion-weighted MR spectroscopy might be a well-suited technique that is being actively developed and validated to disentangle neuronal and glial mechanisms during development in the deep white matter structures ${ }^{103}$. It will be important in the future to conduct similar studies in larger samples, relating the microstructural findings to neurodevelopmental disorder and psychiatric risk - preferably using a longitudinal design to better understand these risks. 


\subsubsection{Strengths and limitations}

A distinguishing feature of the current study is the image contrast enhancement gained from using the unique ultra-strong gradients of the Connectom 3T scanner. The strong gradients allow a given diffusion encoding to be achieved over a shorter time-interval. In this way, diffusion scan echo times were shortened significantly, thereby reducing T2-related signal loss, and boosting SNR by $40-70 \%$ in comparison with clinical MRI scanners. This increased the statistical power of the study to detect group differences; in part compensating for the low $N$ associated with the 22q11.2DS participant group. In addition, recruiting children with 22q11.2DS enables us to investigate the neurodevelopmental pathways based on genotype, thus mitigating some of the difficulties with genetic and environmental heterogeneity in clinically ascertained groups such as ASD or ADHD. Overall, efforts to maximize contrast sensitivity of dMRI in both typically developing children and children with 22q11.2DS allowed for the investigation of microstructural hypo-connectivity in brain white matter.

Children with 22q11.2DS are harder to recruit than typically developing children due to the relative rarity of the syndrome and the frequency of MRI contraindications, e.g., history of cardiac surgery. However, despite the relatively low $N$ compared to larger-scale studies carried out across multiple sites, we observed similar effect sizes in diffusion metrics between 22q11.2DS and ASM controls. The enhanced sensitivity of our results was in part conferred by the Connectom, where high- $b$ contrast acts as a filter for axons and glial processes, versus DTI (using low $b$ ) where microscopic differences are often masked. In addition, children with 22q11.2DS and the ASM group 
were within a narrow age-range (11.8 to 14.9$)$, limiting the influence of variance due to known age-effects. Even so, the effect sizes reported for children with 22q11.2DS are still susceptible to magnitude and sign errors, and should be interpreted cautiously ${ }^{104}$.

The current length of the image acquisition was demanding for children, particularly those with $22 \mathrm{q} 11.2 \mathrm{DS}$, although this was partly mitigated by providing the opportunity to acclimatize to the scanning procedure in a mock-scanner. Despite the richness of the protocol, certain recent developments in biophysical modeling, e.g., axon diameter mapping, could not be applied to these data, as the diffusion times were insufficiently short to resolve different diameters. As such, there was no direct in vivo quantification of axon diameter which would have further strengthened the validation of disrupted axon morphology (see Suppl. Fig. S4 on sensitivity to axon diameters). Despite earlier success in using the Connectom scanner to quantify axon diameters in vivo $55,78,105$, these measurements require extensive scan times that are currently not compatible with the time constraints of scanning children. Characterising axon diameter distributions in this cohort would either require a dedicated study, faster imaging techniques that maximise participant comfort, or the incorporation of motion correction strategies that do not rely on image-registration based approaches 106,107 . It would also be interesting to include myelin-sensitive contrasts, e.g. magnetization transfer imaging, as this information is orthogonal to dMRI and has been shown to be sensitive to both both typical and atypical development ${ }^{108,109}$. 


\section{Conclusions}

In summary, our findings provide support that widespread differences in white matter volumes and microstructure are related to disruptions in axonal morphology in children with 22Q11.2DS. Interestingly, the dMRI fingerprint of 22q11.2DS children was most similar in terms of biophysical principal components to those of older children from the TD cohort. Similar findings were also reported in previously published large-scale dMRI studies (i.e., low-b DTI). By exploiting the unique hardware used in this study, we extend the interpretation of dMRI signal beyond microstructural sensitivity towards cellular specificity. We demonstrate evidence for hypo-connectivity in 22Q11.2DS, a hypothesis put forward in a preclinical setting, but for the first time observed in vivo. This is in contrast to new insights in TD, where glial processes are implicated in observed age-effects in white matter microstructure. The sensitivity and specificity provided by the combination of high- $b \mathrm{dMRI}$ and biophysical modelling highlights the discriminatory power of this approach for investigating mechanistic features underlying both typical and atypical developmental populations.

\section{Acknowledgments}

The authors would like to thank the families that participated in this study for their generous contributions. We would like to thank Chantal Tax, Maxime Chamberland and the rest of the CUBRIC Kids team for helpful discussions and advice regarding neuroimaging in the early stage of this project. We would also like to thank John Evans and Umesh Rudrapatna for scanning support and 
Greg Parker for development of the image processing pipeline.

ER was supported by the Marshall-Sherfield postdoctoral fellowship during this work and is now supported by a NIH fellowship (NICHD / 1F32HD103313-01). JV is supported by NIH (NINDS / R01 NS088040). RAK was supported by the UK Medical Research Council SUAG/047 G101400. JD was supported by a Wellcome Trust Clinical Research Training Fellowship (102003/Z/13/Z) during this work and is currently supported by a Wales Clinical Academic Track Fellowship. MvdB is supported by funding from the MRC (MR/T033045/1), NIMH (U01 MH119738-01) and Wellcome Trust ISSF. DKJ is supported by a Wellcome Trust Investigator Award (096646/Z/11/Z) and a Wellcome Trust Strategic Award (104943/Z/14/Z). The Connectom data were acquired at the UK National Facility for in vivo MR Imaging of Human Tissue Microstructure funded by the EPSRC (grant EP/M029778/1), and The Wolfson Foundation. Research was partially performed at the Center of Advanced Imaging Innovation and Research (CAI2R, www.cai2r.net), an NIBIB Biomedical Technology Resource Center (NIH P41 EB017183).

\section{Conflict of Interest}

The authors declare no conflict of interest.

\section{Supplementary Material}

Precision of diffusion metrics The precision of the individual diffusion metrics was evaluated using a Monte-Carlo simulation with 100,000 trials in which noise was added to synthetic data. The 
synthetic data were generated using the biophysical Standard Model (BSM) with realistic ground truth parameters, based on our data, while mimicking the acquisition settings of the study. The noise level was chosen to match the signal-to-noise ratio of the data itself. For each noise realization, all diffusion metrics were estimated and the variability across the different realizations are shown in Suppl.Fig S2. The coefficients of variation are 0.53, 0.82, 1.68, 1.09, 2.88, 3.42, 7.07, $0.41,1.73,1.58,2.10,2.93, .330,8.68,6.07$, and $1.99 \%$ for FA, $\stackrel{\circ}{S}_{\mu}(b=6000), \stackrel{\circ}{S}_{\sigma}(b=500)$, $\stackrel{\circ}{S}_{\sigma}(b=6000), \bar{D}, D_{\perp}, D_{\|}, \stackrel{\circ}{S}_{\mu}(b=500), \bar{K}, K_{\perp}, K_{\|}, f, D_{c}, D_{e}^{\|}, D_{e}^{\perp}$, and $\kappa$, respectively. The kurtosis metrics and, particularly, the compartmental diffusivities of BSM have poor performance in terms of precision. Therefore, we applied smoothing and along-tract analysis to improve the precision of DKI and BSM, respectively. However, the sensitivity of the compartmental diffusivities $D_{c}, D_{e}^{\|}, D_{e}^{\perp}$ is intrinsically challenged by the low precision of the estimator.

Tract Volume Quantification To evaluate the reproducibliity of the tract volume quantification, we re-analyzed test/retest data of five healthy volunteers that were collected during two scanning sessions with exactly the same imaging protocol on the Siemens Connectom 3T MR scanner. For each volunteer, the two test-retest scanning sessions were performed on the same day interleaved by a short break. In both sessions, subjects were re-positioned by the same operator. Despite some minor changes in imaging protocol in comparison to the study-specific data, fiber tracking and automated segmentation was performed on diffusion-weighted images with $b=6000 \mathrm{~s} / \mathrm{mm}^{2}$.

An overview of the most relevant scan parameters: $\mathrm{TR} / \mathrm{TE}=3500 / 66 \mathrm{~ms}$, imaging matrix: $88 \times 88$, voxel dimensions: $2.5 \times 2.5 \times 2.5 \mathrm{~mm}^{3}$, scan acceleration using SMS=2 and GRAPPA 
$(R=2)$.

The inter- and intra-subject variabilty of the estimated volume of all eleven tracts was computed. The variability served as an input to compute the minimally required effect size that could be detected with statistical significance in our study. For this power analysis, we used $G^{\star}$ Power. In Fig. S3, we show the minimally required effect size as a function of the tract volume for each tract. The technique itself lacks sensitivity to detect subtle effects, but is useful for highlighting changes in tract volume of more than $10-20 \%$.

Sensitivity to the axon morphology Even when deploying the exceptionally strong gradients of the Siemens Connectom 3T scanner, the diffusion-weighted signal attenuation perpendicular to a micrometer-thin axons is weak ${ }^{110}$. Aside from the low sensitivity, our study lacks data with changing diffusion time and/or additional ultra-high $b$-values to enable the quantification of the axon diameter directly ${ }^{55}$. Nonetheless, various diffusion parameters have previously been shown to correlate with the axon diameter, e.g. $D_{\perp}{ }^{83,111}$.

In our study, we maximized the direct sensitivity of dMRI to differences in axon diameter by evaluating the spherical mean at a high $b$-value, while minimizing the gradient duration $\delta=7 \mathrm{~ms}$ and diffusion time $\Delta=23.3 \mathrm{~ms}$. As a result, $\stackrel{\circ}{S}_{\mu}\left(b=6000 \mathrm{~s} / \mathrm{mm}^{2}\right)$ correlates strongly with $\frac{f}{D_{c}} S_{\perp}^{\text {Axon }}(r)$, with $S_{\perp}^{\text {Axon }}(r)$ the radial signal attenuation inside a cylindrical axon with radius $r$. 
Vangelderen1994 demonstrated that

$$
\begin{aligned}
\ln S_{\perp}^{\text {Axon }}(r) & =-\frac{2 q^{2} r^{4}}{D_{0}} \sum_{m=1}^{\infty} \frac{t_{c}}{\alpha_{m}^{6}\left(\alpha_{m}^{2}-1\right)} \\
& {\left[2 \alpha_{m}^{2} \frac{\delta}{t_{c}}-2+2 e^{-\alpha_{m}^{2} \delta / t_{c}}+2 e^{-\alpha_{m}^{2} \Delta / t_{c}}-e^{-\alpha_{m}^{2}(\Delta-\delta) / t_{c}}\right.} \\
& \left.-e^{-\alpha_{m}^{2}(\Delta+\delta) / t_{c}}\right]+\mathcal{O}\left(q^{4}\right)
\end{aligned}
$$

where $q=\gamma G$ is the diffusion-weighting wave vector with $\gamma$ the gyromagnetic ratio for protons and $G$ the gradient strength. Furthermore, $D_{0}$ is the diffusivity of the axoplasm, $\alpha_{m}$ is the $m^{\text {th }}$ root of $\mathrm{d} J_{1}(\alpha) / \mathrm{d} \alpha=0$, and $J_{1}(\alpha)$ is the Bessel function of the first kind. Here, $t_{c}=r^{2} / D_{0}$ is the diffusion time across the axon; see Suppl. Fig. S3.

The reduction of axon diameter specifically will directly result in a higher $\stackrel{\circ}{S}_{\mu}(b=6000)$. This effect is mainly prominent if large axons are affected, but weakens significantly at lower $b$ values, in part because the extra-cellular signal is not fully-suppressed and is likely to mask the effects at $b<6000 \mathrm{~s} / \mathrm{mm}^{2}{ }^{112}$. The unique gradient strength of the scanner used here was critical to our exploration of the axonal morphology, because a similar sensitivity cannot be achieved on clinical MR scanners with gradient strengths up to $80 \mathrm{mT} / \mathrm{m}$, even for the same $b$-values, Fig. S3.

The biophysical interpretation of the BPC Here we provide a more in-depth justification for our biophysical interpretation of the first BPC: intracellular signal.

The first BPC is comprised of FA, $\stackrel{\circ}{S}_{\sigma}$ (low and high $b$ ), and $\stackrel{\circ}{S}_{\mu}$ (high $b$ only). On its own, FA is not specific to any particular microstructural property, e.g., it might reflect differences in orientational dispersion or cellular packing, or both, and this has been the Achilles heel of diffusion 
MR since its inception decades ago $23,25,26,113$.

Evidence of strong co-variance with more specific and interpretable metrics, such as $\stackrel{\circ}{\sigma}_{\sigma}$ and $\stackrel{\circ}{S}_{\mu}$ obtained at high $b$-values, allows for a more specific interpretation. As high $b$-value diffusionweighting suppresses the signal from the extracellular matrix (on account of the higher mobility in this space), the specificity to the intracellular (i.e., neuronal or glial) signal fraction increases ${ }^{112}$.

At high $b$-values, both $\stackrel{\circ}{S}_{\sigma}$ and $\stackrel{\circ}{S}_{\mu}$ are sensitive to the intracellular signal fraction, whereas only $\stackrel{S}{S}_{\sigma}$ is sensitive to orientational dispersion. Therefore, if orientational dispersion had been a dominant biophysical feature, then $\stackrel{\circ}{S}_{\sigma}$ would have reflected this, and $\stackrel{\circ}{S}_{\sigma}$ and $\stackrel{\circ}{S}_{\mu}$ would not have loaded onto the same representative component. The fact that these measures do, indeed, covary so strongly allows us to conclude that it is changes in the intracellular signal fraction that drive age-dependent increases in FA, rather than changes in orientational alignment - a process typically lumped into descriptions of FA. Furthermore, this finding is reinforced by concomitant higher values in BSM parameter $f$, the intracellular signal fraction.

\section{References}

1. Koshiyama, D. et al. White matter microstructural alterations across four major psychiatric disorders: mega-analysis study in 2937 individuals. Molecular Psychiatry 25, 883-895 (2020)

2. Smoller, J. W. et al. Psychiatric genetics and the structure of psychopathology. Molecular Psychiatry 24, 409-420 (2019) 
3. Doherty, J. L. \& Owen, M. J. Genomic insights into the overlap between psychiatric disorders: Implications for research and clinical practice. Genome Medicine 6 (2014)

4. McDonald-McGinn, D. M. et al. 22Q11.2 Deletion Syndrome. Nat Rev Dis Primers 621-626 (2015)

5. Schneider, M. et al. Psychiatric disorders from childhood to adulthood in $22 \mathrm{q} 11.2$ deletion syndrome: results from the international consortium on brain and behavior in $22 \mathrm{q} 11.2$ deletion syndrome. American Journal of Psychiatry 171, 627-639 (2014)

6. Eaton, C. B. et al. Epilepsy and seizures in young people with 22q11.2 deletion syndrome: prevalence and links with other neurodevelopmental disorders. Epilepsia 60, 818-829 (2019)

7. Zinkstok, J. R. et al. Neurobiological perspective of 22q11.2 deletion syndrome. The Lancet Psychiatry 6, 951-960 (2019)

8. Cunningham, A. C. et al. Developmental coordination disorder, psychopathology and IQ in 22q11.2 deletion syndrome. British Journal of Psychiatry 212, 27-33 (2018)

9. Monks, S. et al. Further evidence for high rates of schizophrenia in 22q11.2 deletion syndrome. Schizophrenia Research 153, 231-236 (2014)

10. Meechan, D. W. et al. Modeling a model: Mouse genetics, 22q11.2 Deletion Syndrome, and disorders of cortical circuit development. Progress in Neurobiology 130, 1-28 (2015)

11. Fernandez, A. et al. Mitochondrial dysfunction leads to cortical under-connectivity and cognitive impairment. Neuron 102, 1127-1142 (2019) 
12. Mukai, J. et al. Molecular substrates of altered axonal growth and brain connectivity in a mouse model of schizophrenia. Neuron 86, 680-695 (2015)

13. Sigurdsson, T., Stark, K. L., Karayiorgou, M., Gogos, J. A. \& Gordon, J. A. Impaired hippocampal-prefrontal synchrony in a genetic mouse model of schizophrenia. Nature 464, $763-767(2010)$

14. Buzsaki, G. et al. Neuronal oscillations in cortical networks. Science 304, 1926-1930 (2004)

15. Pajevic, S., Basser, P. J. \& Fields, R. D. Role of myelin plasticity in oscillations and synchrony of neuronal activity. Neuroscience 276, 135-147 (2014)

16. Haroutunian, V. et al. Myelination, oligodendrocytes, and serious mental illness. Glia 1-34 (2014)

17. Perrin, J. S. et al. Growth of white matter in the adolescent brain: role of testosterone and androgen receptor. Journal of Neuroscience 28, 9519-9524 (2008)

18. Yakovlev, P. I. \& Lecours, A.-R. The myelogenetic cycles of regional maturation of the brain. In Regional Development of Brain in Early Life, 3-70 (1967)

19. Mills, K. L., Lalonde, F., Clasen, L. S., Giedd, J. N. \& Blakemore, S.-J. Developmental changes in the structure of the social brain in late childhood and adolescence. Social Cognitive and Affective Neuroscience 9, 123-131 (2014)

20. Lebel, C., Treit, S. \& Beaulieu, C. A review of diffusion MRI of typical white matter development from early childhood to young adulthood. NMR in Biomedicine 1-23 (2017) 
21. Rogdaki, M. et al. Magnitude and heterogeneity of brain structural abnormalities in $22 \mathrm{q} 11.2$ deletion syndrome: a meta-analysis. Molecular Psychiatry (2020)

22. Einstein, A. Uber die von der molekularkinetischen Theorie der Warme geforderte Bewegumg von in ruhenden Flussigkeiten suspendierten Teilchen. Annalen der physik 322, 549-560 (1905)

23. Le Bihan, D. et al. MR imaging of intravoxel incoherent motions: application to diffusion and perfusion in neurologic disorders. Radiology 161, 401-407 (1986)

24. Callaghan, P. T., Jolley, K. W. \& Lelievre, J. Diffusion of water in the endosperm tissue of wheat grains as studied by pulsed field gradient nuclear magnetic resonance. Biophysical Journal 28, 133-141 (1979)

25. Tanner, J. Self diffusion of water in frog muscle. Biophysical Journal 28, 107-116 (1979)

26. Beaulieu, C. The basis of anisotropic water diffusion in the nervous system - A technical review. NMR in Biomedicine 15, 435-455 (2002)

27. McNab, J. A. et al. The Human Connectome Project and beyond: initial applications of 300mT/m gradients. NeuroImage 80, 234-245 (2013)

28. Jones, D. K. et al. Microstructural imaging of the human brain with a 'super-scanner': 10 key advantages of ultra-strong gradients for diffusion MRI. NeuroImage 182, 8-38 (2018)

29. Novikov, D. S., Kiselev, V. G. \& Jespersen, S. N. On modeling. Magnetic Resonance in Medicine 79, 3172-3193 (2018) 
30. Niarchou, M. et al. Psychopathology and cognition in children with 22q11.2 deletion syndrome. British Journal of Psychiatry 204, 46-54 (2014)

31. Chawner, S. J. et al. Childhood cognitive development in 22q11.2 deletion syndrome: casecontrol study. The British Journal of Psychiatry 211, 223-230 (2017)

32. Morrison, S. et al. Cognitive deficits in childhood, adolescence and adulthood in 22q11.2 deletion syndrome and association with psychopathology. Translational Psychiatry 10 (2020)

33. Wechsler, D. Wechsler Abbreviated Scale of Intelligence (The Psychological Corporation, San Antonio, TX, 1999)

34. Berument, S. K., Rutter, M., Lord, C., Pickles, A. \& Bailey, A. Autism screening questionnaire: diagnostic validity. British Journal of Psychiatry 175, 444-451 (1999)

35. Angold, A. et al. Development of a short questionnaire for use in epidemiological studies of depression in children and adolescents. International Journal of Methods in Psychiatric Research 5, 237-249 (1995)

36. Ottman, R. et al. Validation of a brief screening instrument for the ascertainment of epilepsy. Epilepsia 51, 191-197 (2010)

37. De Bie, H. M. et al. Preparing children with a mock scanner training protocol results in high quality structural and functional MRI scans. European Journal of Pediatrics 169, 1079-1085 (2010) 
38. Jones, D. K., Simmons, A., Williams, S. C. \& Horsfield, M. A. Non-invasive assessment of axonal fiber connectivity in the human brain via diffusion tensor MRI. Magnetic Resonance in Medicine 42, 37-41 (1999)

39. Veraart, J., Fieremans, E. \& Novikov, D. S. Diffusion MRI noise mapping using random matrix theory. Magnetic Resonance in Medicine 76, 1582-1593 (2016)

40. Vos, S. B. et al. The importance of correcting for signal drift in diffusion MRI. Magnetic Resonance in Medicine 77, 285-299 (2017)

41. Andersson, J. L. \& Sotiropoulos, S. N. An integrated approach to correction for off-resonance effects and subject movement in diffusion MR imaging. NeuroImage 125, 1063-1078 (2016)

42. Kellner, E., Dhital, B., Kiselev, V. G. \& Reisert, M. Gibbs-ringing artifact removal based on local subvoxel-shifts. Magnetic Resonance in Medicine 76, 1574-1581 (2016)

43. Sairanen, V., Leemans, A. \& Tax, C. M. Fast and accurate Slicewise OutLIer Detection (SOLID) with informed model estimation for diffusion MRI data. NeuroImage 181, 331$346(2018)$

44. Tournier, J. D. et al. MRtrix3: A fast, flexible and open software framework for medical image processing and visualisation. NeuroImage 202, 116137 (2019)

45. Jenkinson, M., Beckmann, C. F., Behrens, T. E. J., Woolrich, M. W. \& Smith, S. M. FSL. NeuroImage 62, 782-790 (2012) 
46. Stanisz, G. J., Szafer, A., Wright, G. A. \& Henkelman, R. M. An analytical model of restricted diffusion in bovine optic nerve. Magnetic Resonance in Medicine 37, 103-111 (1997)

47. Jespersen, S. N., Kroenke, C. D., Østergaard, L., Ackerman, J. J. \& Yablonskiy, D. A. Modeling dendrite density from magnetic resonance diffusion measurements. NeuroImage 34, $1473-1486(2007)$

48. Novikov, D. S., Veraart, J., Jelescu, I. O. \& Fieremans, E. Rotationally-invariant mapping of scalar and orientational metrics of neuronal microstructure with diffusion MRI. NeuroImage $174,518-538(2018)$

49. Basser, P. J. \& Pierpaoli, C. Microstructural and physiological features of tissues elucidated by quantitative-diffusion-tensor MRI. Journal of Magnetic Resonance - Series B 111, 209$219(1996)$

50. Jensen, J. H., Helpern, J. A., Ramani, A., Lu, H. \& Kaczynski, K. Diffusional Kurtosis Imaging: the quantification of non-Gaussian water diffusion by means of magnetic resonance imaging. Magnetic Resonance in Medicine 53, 1432-1440 (2005)

51. Jensen, J. H. \& Helpern, J. A. MRI quantification of non-Gaussian water diffusion by kurtosis analysis. NMR in Biomedicine 23, 698-710 (2010)

52. Veraart, J., Sijbers, J., Sunaert, S., Leemans, A. \& Jeurissen, B. Weighted linear least squares estimation of diffusion MRI parameters: strengths, limitations, and pitfalls. NeuroImage 81, $335-346(2013)$ 
53. Parker, G. J., Schnabel, J. A., Symms, M. R., Werring, D. J. \& Barker, G. J. Nonlinear smoothing for reduction of systematic and random errors in diffusion tensor imaging. Journal of Magnetic Resonance Imaging 11, 702-710 (2000)

54. Mirzaalian, H. et al. Inter-site and inter-scanner diffusion MRI data harmonization. NeuroImage 135, 311-323 (2016)

55. Veraart, J. et al. Noninvasive quantification of axon radii using diffusion MRI. eLife 9, 1-27 (2020)

56. Zhang, Y., Brady, M. \& Smith, S. Segmentation of brain MR images through a hidden Markov random field model and the expectation-maximization algorithm. IEEE Transactions on Medical Imaging 20, 45-57 (2001)

57. Wasserthal, J., Neher, P. F., Hirjak, D. \& Maier-Hein, K. H. Combined tract segmentation and orientation mapping for bundle-specific tractography. Medical Image Analysis 58, 101559 (2019)

58. Calamante, F., Tournier, J. D., Jackson, G. D. \& Connelly, A. Track-density imaging (TDI): super-resolution white matter imaging using whole-brain track-density mapping. NeuroImage 53, 1233-1243 (2010)

59. Jelescu, I. O., Veraart, J., Fieremans, E. \& Novikov, D. S. Degeneracy in model parameter estimation for multi-compartmental diffusion in neuronal tissue. NMR in Biomedicine 29, 33-47 (2016) 
60. Klein, J., Hermann, S., Konrad, O., Hahn, H. K. \& Peitgen, H.-O. Automatic quantification of DTI parameters along fiber bundles. In Bildverarbeitung für die Medizin, 272-276 (2007)

61. Yeatman, J. D., Dougherty, R. F., Myall, N. J., Wandell, B. A. \& Feldman, H. M. Tract profiles of white matter properties: automating fiber-tract quantification. PLoS ONE 7 (2012)

62. Maier-Hein, K. H. The challenge of mapping the human connectome based on diffusion tractography. Nature Communications (2017)

63. R Core Team. R: A language and environment for statistical computing. (2020). URL https://www.r-project.org/

64. Field, A., Miles, J. \& Field, Z. Discovering statistics using R (2012)

65. Genc, S. et al. Impact of b-value on estimates of apparent fibre density. Human Brain Mapping 41, 2583-2595 (2020)

66. Benjamini, Y. \& Hochberg, Y. Controlling the false discovery rate: a practical and powerful approach to multiple testing. Journal of the Royal Statistical Society. 57, 289-300 (1995)

67. Lakens, D. Calculating and reporting effect sizes to facilitate cumulative science: a practical primer for t-tests and ANOVAs. Frontiers in Psychology 4, 1-12 (2013)

68. Giedd, J. N., Castellanos, F. X., Rajapakse, J. C., Vaituzis, A. C. \& Rapoport, J. L. Sexual dimorphism of the developing human brain. Progress in Neuro-Psychopharmacology and Biological Psychiatry 21, 1186-1201 (1997) 
69. Durston, S. et al. Anatomical MRI of the developing human brain: what have we learned? Journal of the American Academy of Child \& Adolescent Psychiatry 40, 1012-1020 (2001)

70. Chamberland, M. et al. Dimensionality reduction of diffusion MRI measures for improved tractometry of the human brain. NeuroImage 200, 89-100 (2019)

71. de Mooij, S. M., Henson, R. N., Waldorp, L. J. \& Kievit, R. A. Age differentiation within gray matter, white matter, and between memory and white matter in an adult life span cohort. Journal of Neuroscience 38, 5826-5836 (2018)

72. van Kesteren, E.-J. \& Kievit, R. Exploratory factor analysis with structured residuals for brain imaging data. Network Neuroscience (2020)

73. Cox, S. R. et al. Ageing and brain white matter structure in 3,513 UK Biobank participants. Nature Communications 7, 1-13 (2016)

74. Villalón-Reina, J. E. et al. Altered white matter microstructure in 22q11.2 deletion syndrome: a multisite diffusion tensor imaging study. Molecular Psychiatry (2019)

75. Lebel, C. \& Beaulieu, C. Longitudinal development of human brain wiring continues from childhood into adulthood. The Journal of Neuroscience 31, 10937-47 (2011)

76. Stuermer, C. A. Rules for retinotectal terminal arborizations in the goldfish optic tectum: a whole-mount study. Journal of Comparative Neurology 229, 214-232 (1984) 
77. Perge, J. A., Koch, K., Miller, R., Sterling, P. \& Balasubramanian, V. How the optic nerve allocates space, energy capacity, and information. The Journal of Neuroscience 29, 79177928 (2009)

78. Drakesmith, M. et al. Estimating axon conduction velocity in vivo from microstructural MRI. NeuroImage 203, 116-186 (2019)

79. Judson, M. C. et al. Decreased axon caliber underlies loss of fiber tract integrity, disproportional reductions in white matter volume, and microcephaly in Angelman syndrome model mice. Journal of Neuroscience 37, 7347-7361 (2017)

80. Wegiel, J. et al. Deficit of corpus callosum axons, reduced axon diameter and decreased area are markers of abnormal development of interhemispheric connections in autistic subjects. Acta Neuropathologica Communications 6, 143 (2018)

81. Zikopoulos, B. \& Barbas, H. Changes in prefrontal axons may disrupt the network in autism. Journal of Neuroscience 30, 14595-14609 (2010)

82. Burcaw, L. M., Fieremans, E. \& Novikov, D. S. Mesoscopic structure of neuronal tracts from time-dependent diffusion. NeuroImage 114, 18-37 (2015)

83. Takahashi, M. et al. Magnetic resonance microimaging of intraaxonal water diffusion in live excised lamprey spinal cord. Proceedings of the National Academy of Sciences 99 (2002)

84. Lamantia, A.-S. \& Rakic, P. Cytological and quantitative characteristics of four cerebral commissures in the rhesus monkey. The Journal of Comparative Neurology 291, 520-537 (1990) 
85. Barres, B. A. The mystery and magic of glia: a perspective on their roles in health and disease. Neuron 60, 430-440 (2008)

86. Oberheim, N. A. et al. Uniquely hominid features of adult human astrocytes. Journal of Neuroscience 29, 3276-3287 (2009)

87. Schwartz, E. D. et al. MRI diffusion coefficients in spinal cord correlate with axon morphometry. NeuroReport 16, 73-76 (2005)

88. Takahashi, M., Ono, J., Harada, K., Maeda, M. \& Hackney, D. B. Diffusional anisotropy in cranial nerves with maturation: quantitative evaluation with diffusion MR imaging in rats. Radiology 216, 881-885 (2000)

89. Paus, T. Growth of white matter in the adolescent brain: myelin or axon? Brain and Cognition 72, 26-35 (2010)

90. Hagmann, P. et al. White matter maturation reshapes structural connectivity in the late developing human brain. Proceedings of the National Academy of Sciences 107, 19067-19072 (2010)

91. Almeida, R. G. \& Lyons, D. A. On myelinated axon plasticity and neuronal circuit formation and function. Journal of Neuroscience 37, 10023-10034 (2017)

92. Costa, A. R., Pinto-Costa, R., Sousa, S. C. \& Sousa, M. M. The regulation of axon diameter: from axonal circumferential contractility to activity-dependent axon swelling. Frontiers in Molecular Neuroscience 11, 1-7 (2018) 
93. Chowen, J. A., Azcoitia, I., Cardona-Gomez, G. P. \& Garcia-Segura, L. M. Sex steroids and the brain: lessons from animal studies. Journal of Pediatric Endocrinology and Metabolism $13,1045-1066(2000)$

94. Maninger, N., Wolkowitz, O. M., Reus, V. I., Epel, E. S. \& Mellon, S. H. Neurobiological and neuropsychiatric effects of dehydroepiandrosterone (DHEA) and DHEA sulfate (DHEAS). Frontiers in Neuroendocrinology 30, 65-91 (2009)

95. Robillard, K. N., Lee, K. M., Chiu, K. B. \& MacLean, A. G. Glial cell morphological and density changes through the lifespan of rhesus macaques. Brain Behavior Immunity 55, 6069 (2016)

96. Sigaard, R. K., Kjær, M. \& Pakkenberg, B. Development of the cell population in the brain white matter of young children. Cerebral Cortex 26, 89-95 (2016)

97. Yoon, H., Walters, G., Paulsen, A. R. \& Scarisbrick, I. A. Astrocyte heterogeneity across the brain and spinal cord occurs developmentally, in adulthood and in response to demyelination. PLoS ONE 12, 1-19 (2017)

98. Hildebrand, C., Remahl, S., Persson, H. \& Bjartmar, C. Myelinated nerve fibres in the CNS. Progress in Neurobiology 40, 319-384 (1993)

99. Bartzokis, G. Neuroglialpharmacology: myelination as a shared mechanism of action of psychotropic treatments. Neuropharmacology 62, 2137-53 (2012)

100. Fields, R. D. White matter in learning, cognition and psychiatric disorders. Trends in Neurosciences $31,361-370(2008)$ 
101. Verkhratsky, A. \& Nedergaard, M. Physiology of astroglia. Physiological Reviews 98, 239389 (2018)

102. Molofsk, A. V. et al. Astrocytes and disease: a neurodevelopmental perspective. Genes and Development 26, 891-907 (2012)

103. Ligneul, C. et al. Diffusion-weighted magnetic resonance spectroscopy enables cell-specific monitoring of astrocyte reactivity in vivo. NeuroImage 191, 457-469 (2019)

104. Gelman, A. \& Carlin, J. Beyond power calculations: assessing Type S (Sign) and Type M (Magnitude) errors. Perspectives on Psychological Science 9, 641-651 (2014)

105. Huang, S. Y. et al. High-gradient diffusion MRI reveals distinct estimates of axon diameter index within different white matter tracts in the in vivo human brain. Brain Structure and Function 225, 1277-1291 (2020)

106. Callaghan, M. F. et al. An evaluation of prospective motion correction (PMC) for high resolution quantitative MRI. Frontiers in Neuroscience 9, 1-9 (2015)

107. Herbst, M., Zahneisen, B., Knowles, B., Zaitsev, M. \& Ernst, T. Prospective motion correction of segmented diffusion weighted EPI. Magnetic Resonance in Medicine 74, 1675-1681 $(2015)$

108. Whitaker, K. J. et al. Adolescence is associated with genomically patterned consolidation of the hubs of the human brain connectome. Proceedings of the National Academy of Sciences $113,201601745(2016)$ 
109. Romero-Garcia, R. et al. Schizotypy-related magnetization of cortex in healthy adolescence Is colocated with expression of schizophrenia-related genes. Biological Psychiatry 248-259 (2020)

110. van Gelderen, P., DesPres, D., van Zijl, P. \& Moonen, C. Evaluation of restricted diffusion in cylinders. Phosphocreatine in rabbit leg muscle. Journal of Magnetic Resonance 103, 255-260 (1994)

111. Barazany, D., Basser, P. J. \& Assaf, Y. In vivo measurement of axon diameter distribution in the corpus callosum of rat brain. Brain 132, 1210-20 (2009)

112. Veraart, J., Fieremans, E. \& Novikov, D. S. On the scaling behavior of water diffusion in human brain white matter. NeuroImage 185, 379-387 (2019)

113. Jones, D. K., Knösche, T. R. \& Turner, R. White matter integrity, fiber count, and other fallacies: the do's and don'ts of diffusion MRI. NeuroImage 73, 239-54 (2013) 
10 Figures 

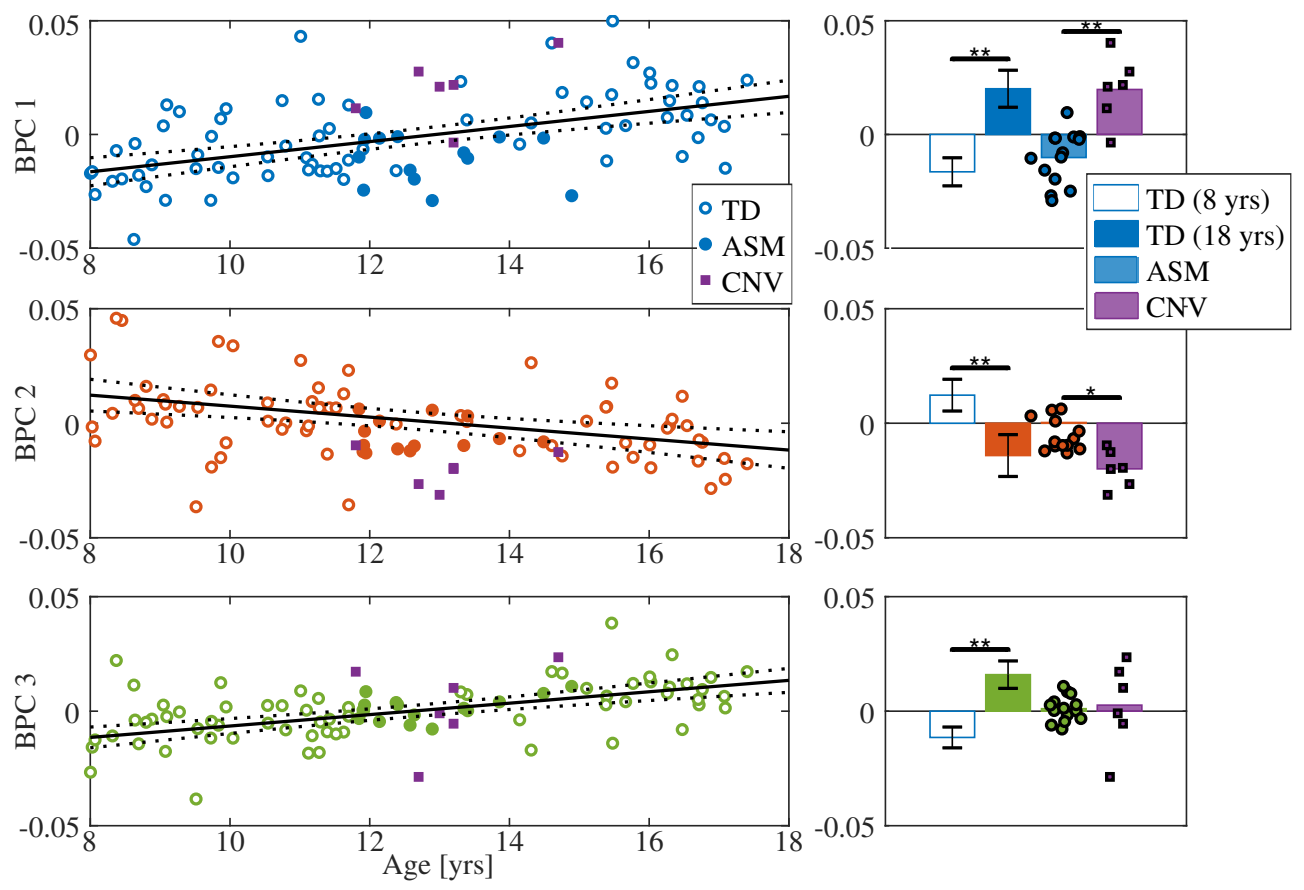

(a) BPC trends

(b) BPC distributions

Figure 1: Age associations with biophysical principal components. BPC1 is intracellular signal (blue), BPC2 is extracellular mobility (red), and BPC3 is tissue complexity (green). A) BPC values are correlated with age in total white matter. Points are highlighted for age- and sex- matched controls (filled circles), and 22q11.2DS participants (filled squares). The black line denotes the linear regression for the TD group only (with dashed 95\% confidence intervals). B) Nominal values are computed for 1 ) estimates from the linear regression for youngest ( 8 year old) and oldest (18 year old) TD participants, and 2) measured BPC scores of ASM and 22q11.2DS participants. Asterisk denotes significance between group means $\left({ }^{*} p<0.05,{ }^{* *} p<0.001\right)$. 


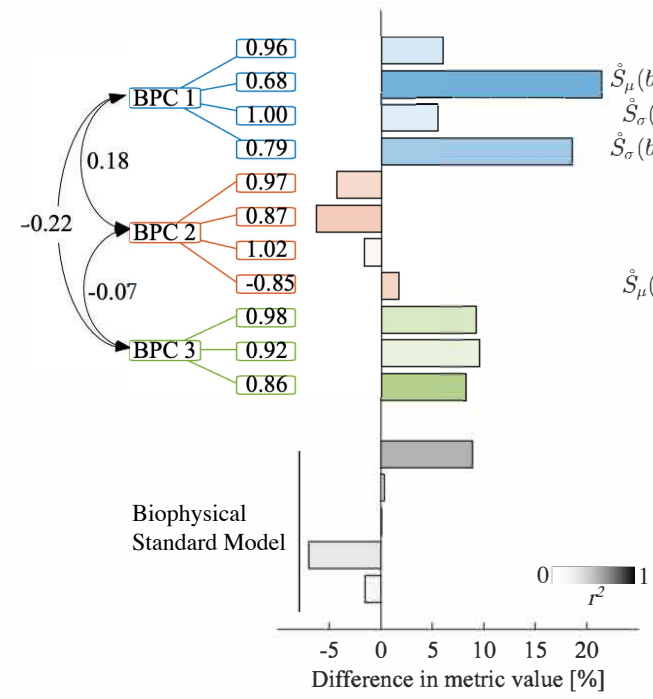

(a) BPC loadings

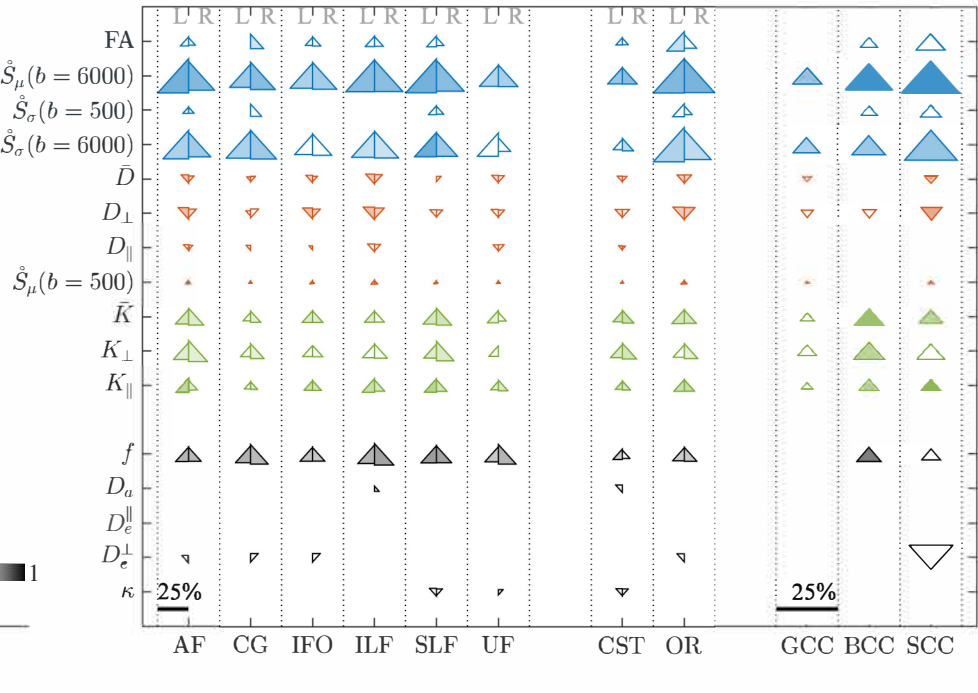

(c) Effect size: individual white matter tracts

Figure 2: dMRI fingerprint of development. Metrics are grouped by BPC1 (blue), BPC2 (red), BPC3 (green), and Biophysical Standard Model parameters (black). (a) The highest metric loadings are shown for each BPC. (b) Bar plots are encoded with linear regression statistics in total white matter. Color intensity is effect size $\left(r^{2}\right)$; bar length is percent difference of metric estimates from the linear regression for youngest ( 8 year old) and oldest (18 year old) TD participants. (c) Triangle plots are the same statistics for individual white matter tracts. Triangles are divided by left (L) and right (R) hemisphere for association and projection tracts. Commissural tracts span both hemispheres and are not divided. Percent differences are denoted by the scale bar, reaching a maximum of $25 \%$ difference. Triangle orientation, i.e., up or down, denotes positive or negative associations with age, respectively. Triangles are omitted that do not survive statistical significance. 

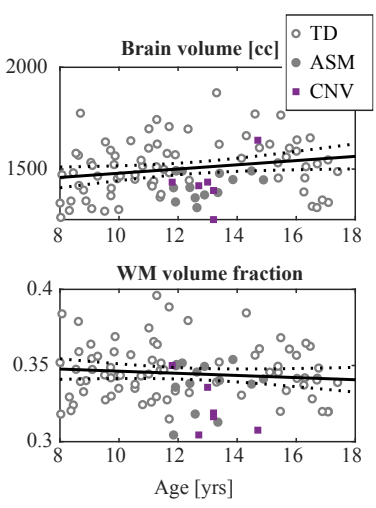

(a) Tissue volume and fraction: data trend
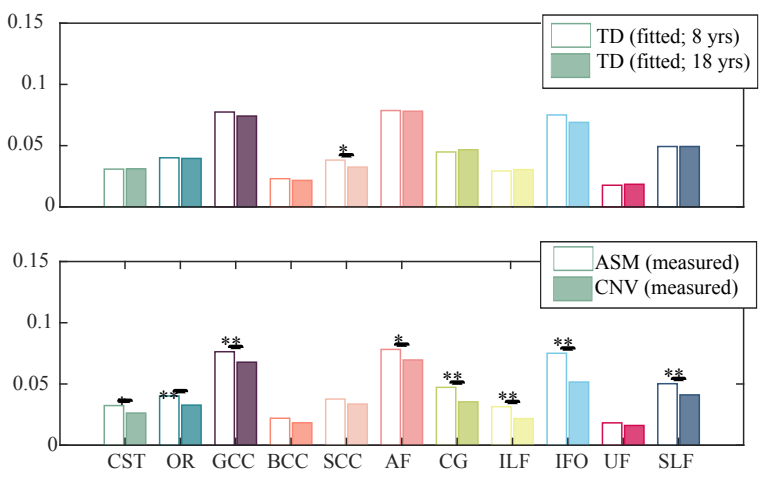

(c)Tract-specific WM volume differences

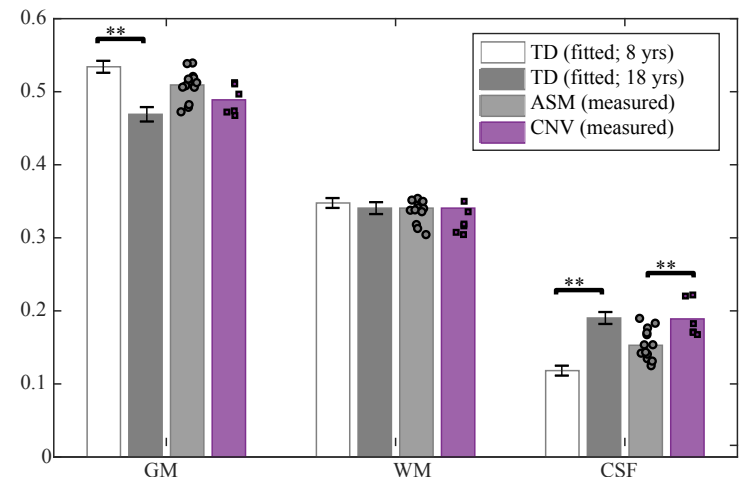

(b) Tissue volume fraction

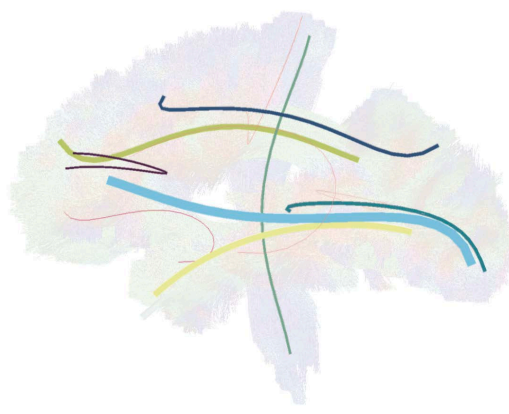

(d) Spatial distribution of WM volume differences

Figure 3: Whole brain and tract volume fractions in TD and 22q11.2DS participants. (a) Total brain volume and gray matter (GM), white matter (WM), and cerebrospinal fluid (CSF) volume fractions are correlated with age. Points are highlighted for ASM (filled circles) and 22q11.2DS participants (filled squares). The black line denotes the linear regression line for the TD group only (with dashed 95\% confidence intervals). (b) Nominal differences in GM, WM, and CSF are shown for volume fraction estimates from the linear regression for youngest (8 year old) and oldest (18 year old) TD participants. In addition, measured volume fractions are shown for ASM and 22q11.2DS participants (data points overlaid on bar). (c) Individual WM tract volume fractions are shown for (top) youngest (8 year old) and oldest (18 year old) TD, and (bottom) ASM and 22q11.2DS participants. (d) Percent differences in WM tract volume fractions for ASM and 22q11.2DS participants are overlaid on a single subject's tractogram. The radius of each tract 50 'tube' reflects the percent difference between groups (range from 0-35\%). Tract colors match panel C. Asterisk denotes significance between groups $\left({ }^{*} p<0.05,{ }^{* *} p<0.001\right)$. 


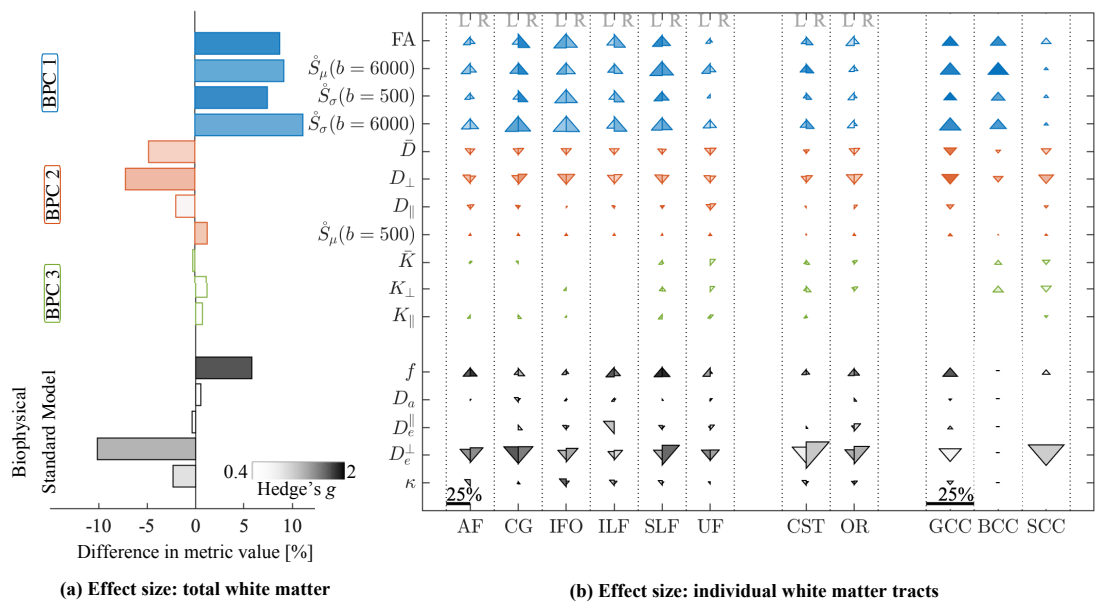

Figure 4: dMRI fingerprint of 22q11.2DS. Metrics are grouped by BPC1 (blue), BPC2 (red), BPC3 (green), and Biophysical Standard Model parameters (black). (a) Bar plots are encoded by mean difference and effect size in total white matter. Color intensity is effect size (Hedge's $g$ ); bar length is percent difference between average metric values for ASM and 22q11.2DS participants. (b) Triangle plots are the same statistics for individual white matter tracts. Triangles are divided by left $(\mathrm{L})$ and right $(\mathrm{R})$ hemisphere for association and projection tracts. Commissural tracts span both hemispheres and are not divided. Percent differences are denoted by the scale bar, reaching a maximum of $25 \%$ difference. Triangles pointed up indicate a higher metric values for 22q11.2DS relative to ASM (and vice versa for down). Data from BCC is not shown for the Biophysical Standard Model parameters due to lack of robustness in the along-tract profiling for that particular tract (marked as -). Triangles are omitted for low effect sizes, Hedge's $g<0.2$, and blank for Hedge's $g<0.4$. 


\section{Tables}

\section{TD children}

\begin{tabular}{|c|c|c|}
\hline Sex & $N$ & Age \\
\hline $\mathrm{M}$ & 43 & $11.8(2.6)$ \\
$\mathrm{F}$ & 49 & $12.9(3.1)$ \\
\hline
\end{tabular}

\section{Age- and sex-matched TD children}

\begin{tabular}{|c|c|c|c|}
\hline Sex & $N$ & Age & $\mathrm{IQ}^{\star}$ \\
\hline $\mathrm{F}$ & 14 & $12.9(1.0)$ & $101.8(11.2)$ \\
\hline
\end{tabular}

\section{Children with 22q11.2DS}

\begin{tabular}{|c|c|c|c|c|c|}
\hline Sex & $N$ & Age & IQ & SCQ & CAPA \\
\hline F & 6 & $13.1(1.0)$ & $75.5(9.4)$ & $9.3(4.8)$ & $3.5(5.1)$ \\
\hline
\end{tabular}

Table 1: Demographics of typically developing children and 22q11.2DS participants. Mean and standard deviation are shown for all groups. *Of the age- and sex-matched TD children, 12 participants completed the abbreviated IQ test. $M$ male, $F$ female, $N$ number, $S C Q$ Social Communication Questionnaire, CAPA Child and Adolescent Psychiatric Assessment. 


\begin{tabular}{rrrr}
\hline Metric & BPC1 & BPC2 & BPC3 \\
\hline $\bar{D}$ & -0.10 & 0.97 & 0.09 \\
$D_{\perp}$ & -0.30 & 0.87 & 0.09 \\
$D_{\|}$ & 0.26 & 1.02 & 0.01 \\
$\bar{K}$ & 0.02 & 0.06 & 0.99 \\
$K_{\perp}$ & 0.04 & 0.07 & 0.92 \\
$K_{\|}$ & -0.04 & -0.14 & 0.86 \\
& & & \\
$\stackrel{\circ}{S}_{\mu}(b=500)$ & 0.08 & -0.85 & 0.25 \\
$\stackrel{\circ}{S}_{\mu}(b=6000)$ & 0.68 & -0.11 & 0.41 \\
$\stackrel{\circ}{S}_{\sigma}(b=500)$ & 1.00 & 0.13 & -0.03 \\
$\stackrel{\circ}{S}_{\sigma}(b=6000)$ & 0.79 & -0.10 & 0.28 \\
\hline & & &
\end{tabular}

Table 2: Standardized factor loadings for total white matter. FA, fractional anisotropy; $\bar{D}$, mean diffusivity; $D_{\perp}$, radial diffusivity; $D_{\|}$, axial diffusivity; $\bar{K}$, mean kurtosis; $K_{\perp}$, radial kurtosis; $K_{\|}$, axial kurtosis; $\stackrel{\circ}{S}_{\mu}(b=500)$, spherical mean at low- $b ; \stackrel{\circ}{S}_{\mu}(b=6000)$, spherical mean at high- $b$; $\stackrel{\circ}{S}_{\sigma}(b=500)$, spherical variance at low- $b ; \stackrel{\circ}{S}_{\sigma}(b=6000)$, spherical variance at high- $b$. 


\section{Supplementary figures}
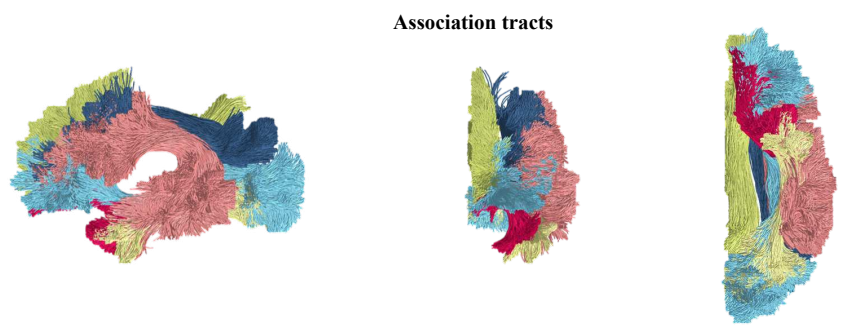

Arcuate fasciculus (AF)

Cingulum $(\mathrm{CG})$

Inf. fronto-occipital fasciculus (IFO)

Inf. longitudinal fasciculus (ILF)

Sup. longitudinal fasciculus (SLF)

Uncinate fasciculus (UF)
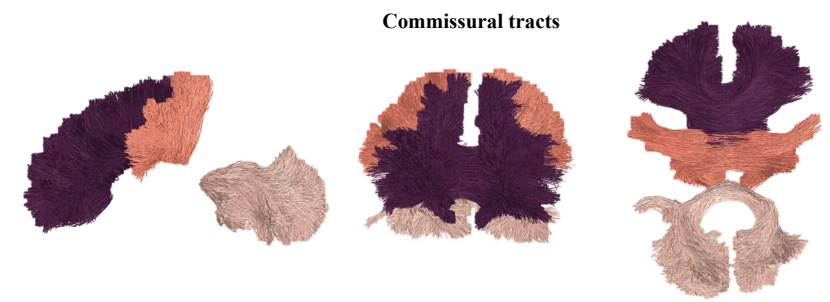

Genu (GCC)

Body (BCC)

Splenium (SCC)

Projection tracts
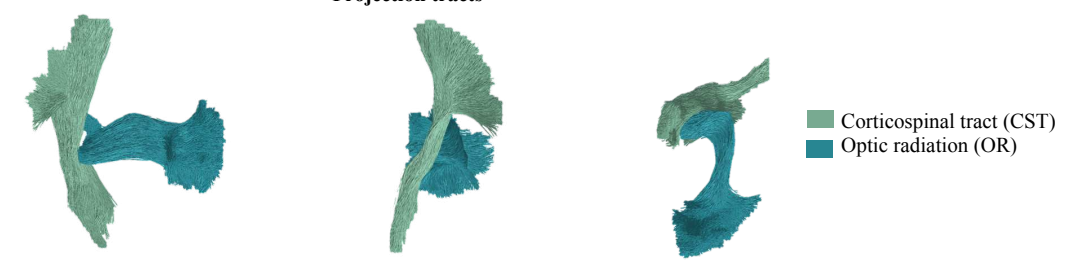

Figure S1: White matter tracts of interest. Eleven fiber tracts extracted using automated segmentation software are shown for an 8-year-old female TD participant. Tracts are grouped by association (top), commissural (middle), and projection (bottom) pathways and shown for left hemisphere. 


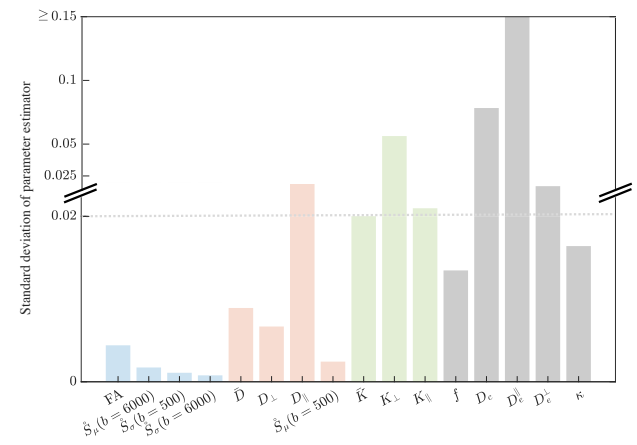

Figure S2: The bar plot shows the standard deviation of the estimator of the various diffusion metrics, as predicted from the simulation experiment. 

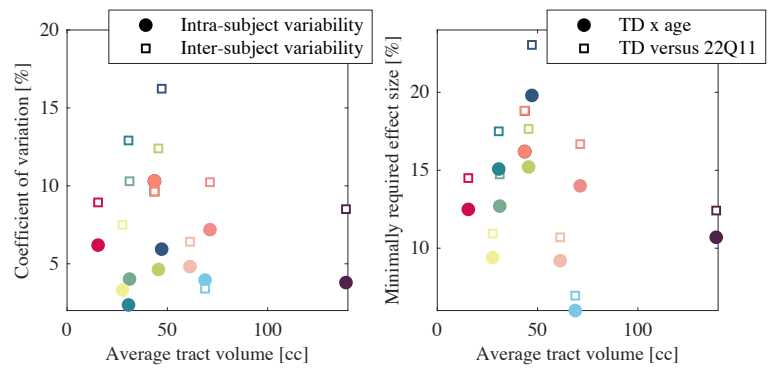

Figure S3: Reproducibility of tract volume quantification. A) The coefficent of variation is shown for intra-subject (closed circle) and inter-subject (open square) tract volume measurements. B) The minimally required effect size is shown for detecting effects in TD (closed circle) and in 22q11.2DS (open square). 


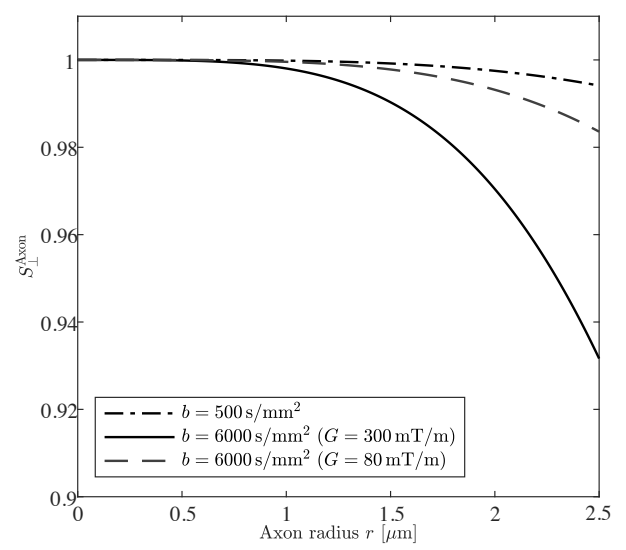

Figure S4: Radial signal decay $S_{\perp}^{\text {Axon }}$ inside a cylindrical axon with radius $r$. The signal decay is evaluated for various $b$-values and gradient strengths to demonstrate the feasibility of the study scanner, i.e. Siemens Connectom 3T with $G=300 \mathrm{mT} / \mathrm{m}$, to sense axon calibers at high $b$-values and compare its performance to state-of-the-art clinical scanners with $G=80 \mathrm{mT} / \mathrm{m}$. 


\section{Figures}
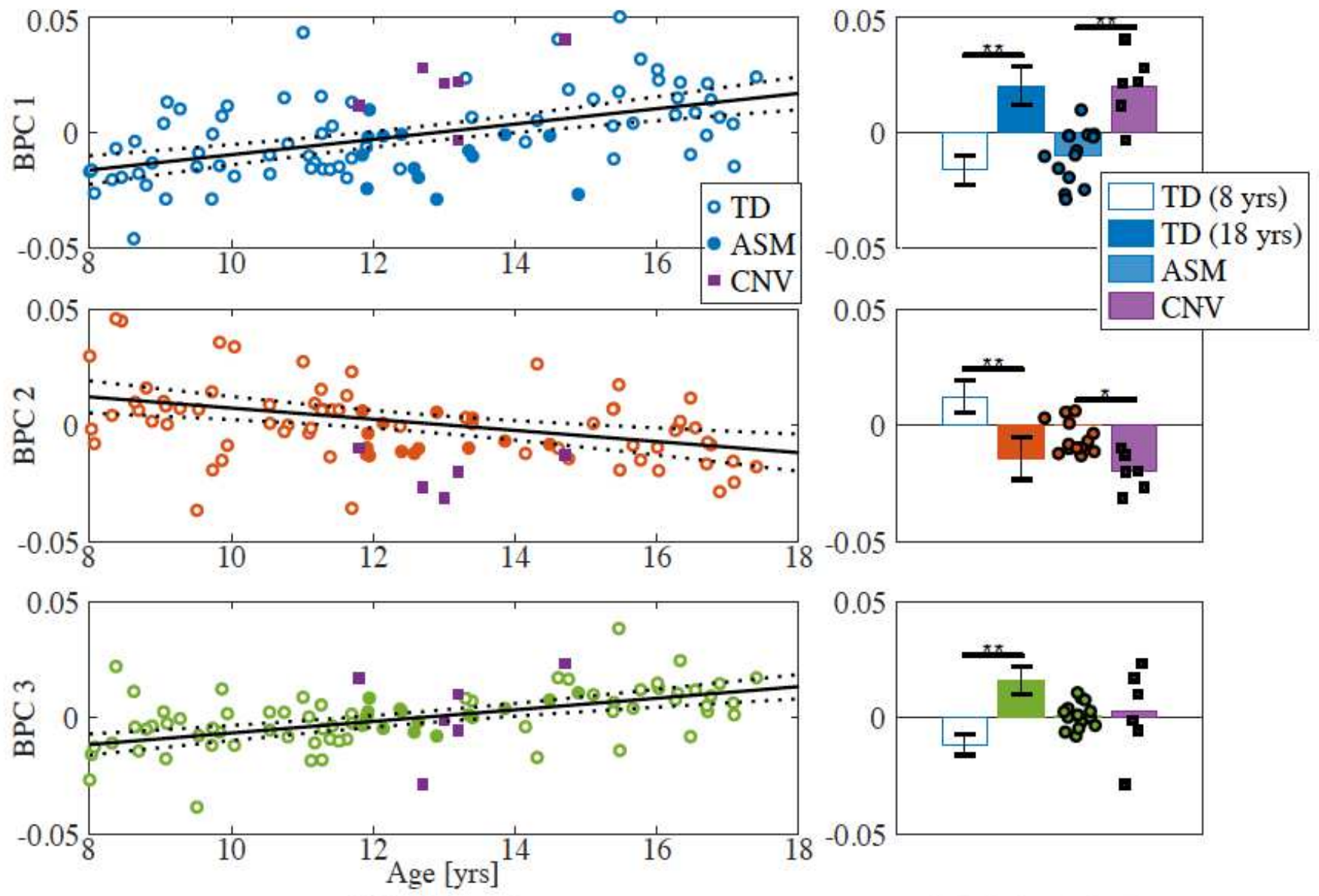

(a) BPC trends

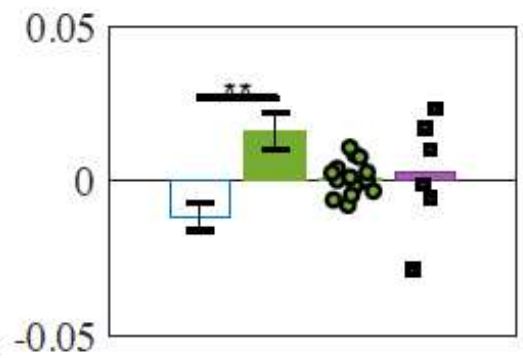

(b) BPC distributions

\section{Figure 1}

Age associations with biophysical principal components. BPC1 is intracellular signal (blue), BPC2 is extracellular mobility (red), and BPC3 is tissue complexity (green). A) BPC values are correlated with age in total white matter. Points are highlighted for age- and sex- matched controls (filled circles), and 22q11.2DS participants (filled squares). The black line denotes the linear regression for the TD group only (with dashed 95\% confidence intervals). B) Nominal values are computed for 1 ) estimates from the linear regression for youngest (8 year old) and oldest (18 year old) TD participants, and 2) measured BPC scores of ASM and 22q11.2DS participants. Asterisk denotes significance between group means ( $\mathbb{p}<<$ $0: 05$, 怄 $p<0: 001)$. 


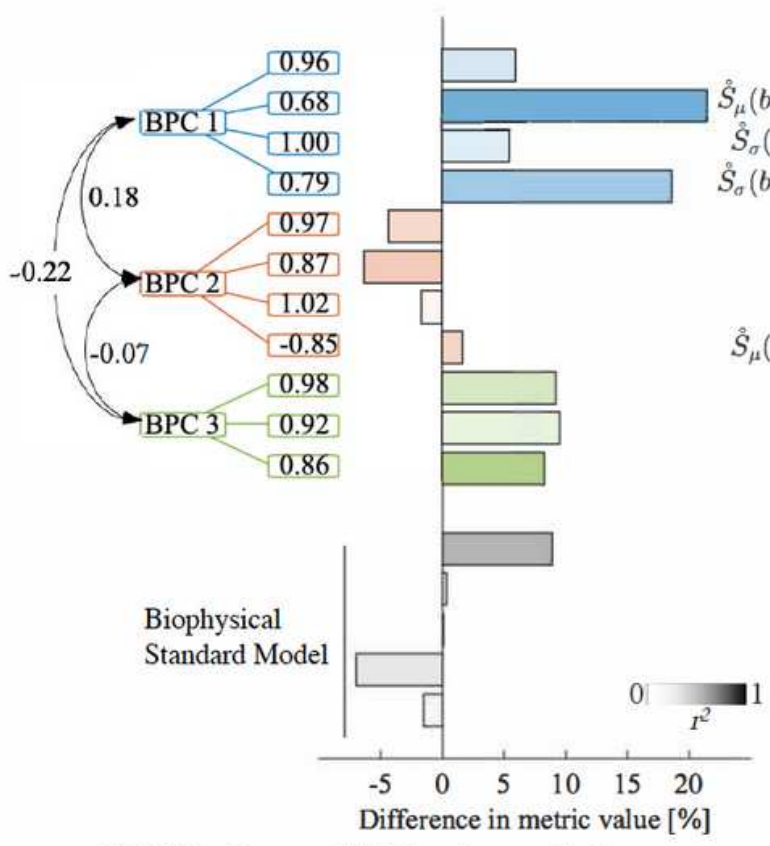

(a) BPC loadings

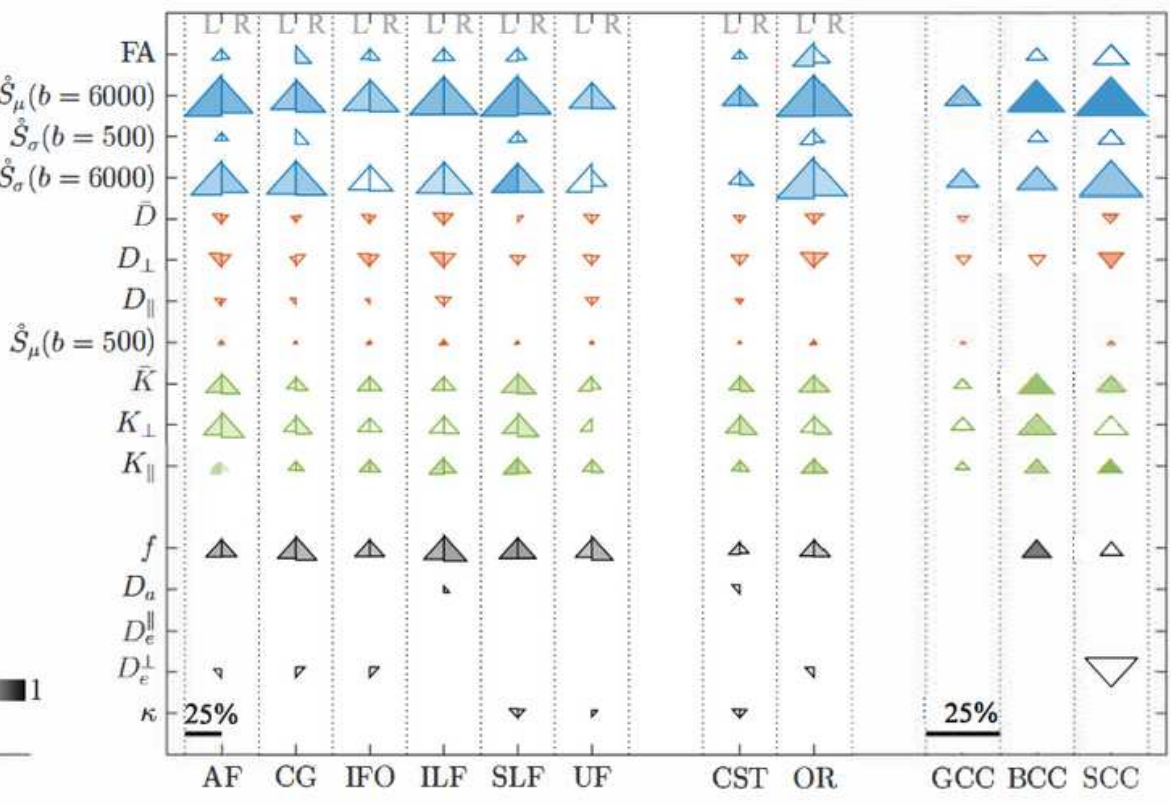

(c) Effect size: individual white matter tracts

\section{Figure 2}

dMRI fingerprint of development. Metrics are grouped by BPC1 (blue), BPC2 (red), BPC3 (green), and Biophysical Standard Model parameters (black). (a) The highest metric loadings are shown for each BPC. (b) Bar plots are encoded with linear regression statistics in total white matter. Color intensity is effect size (r2); bar length is percent difference of metric estimates from the linear regression for youngest (8 year old) and oldest (18 year old) TD participants. (c) Triangle plots are the same statistics for individual white matter tracts. Triangles are divided by left $(\mathrm{L})$ and right $(\mathrm{R})$ hemisphere for association and projection tracts. Commissural tracts span both hemispheres and are not divided. Percent differences are denoted by the scale bar, reaching a maximum of $25 \%$ difference. Triangle orientation, i.e., up or down, denotes positive or negative associations with age, respectively. Triangles are omitted that do not survive statistical significance. 

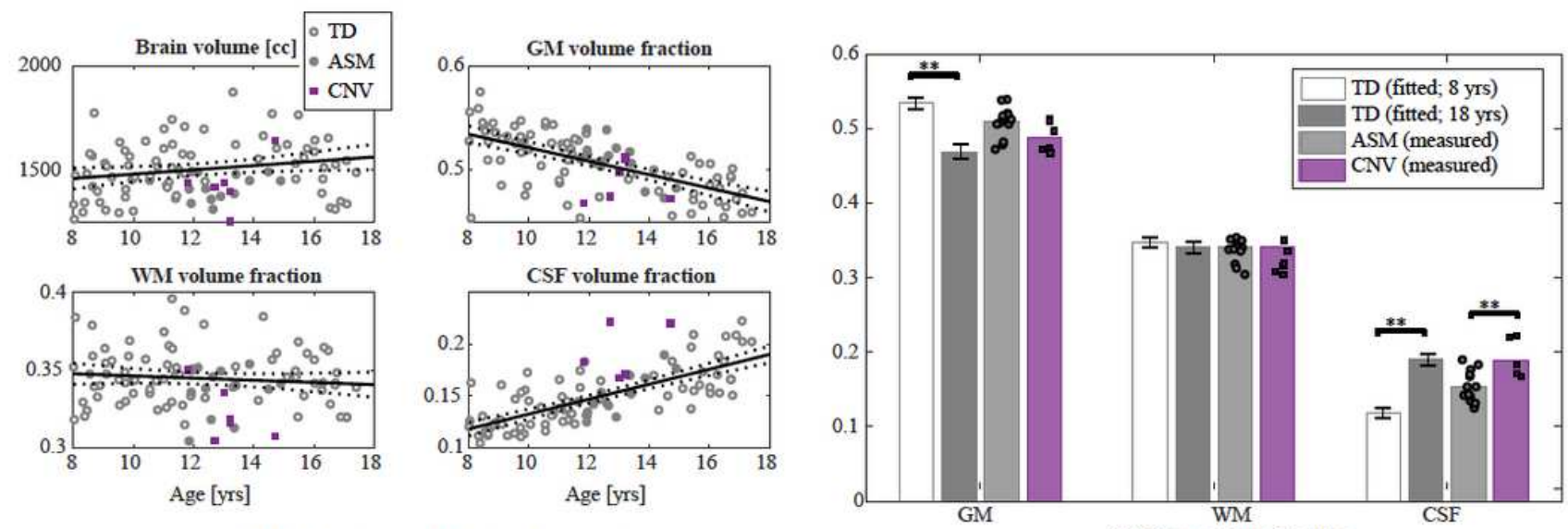

(a) Tissue volume and fraction: data trend
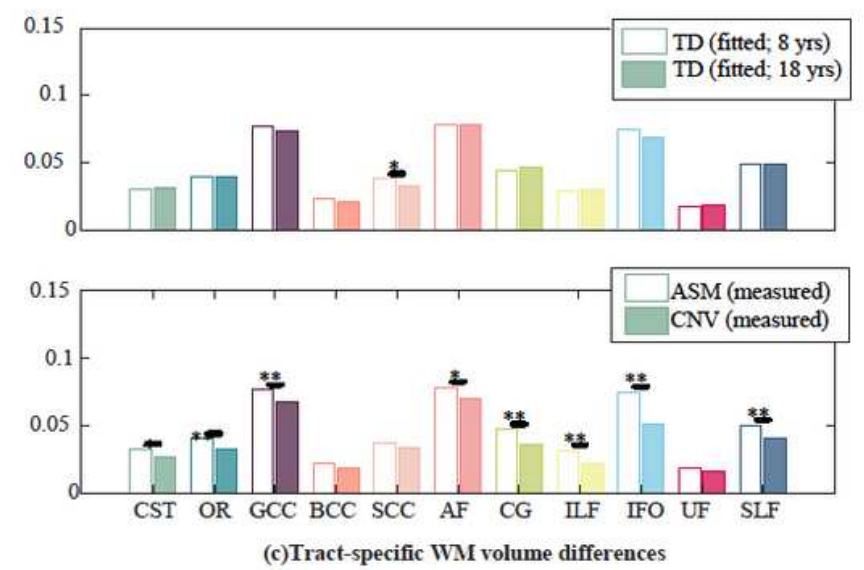

(b) Tissue volume fraction

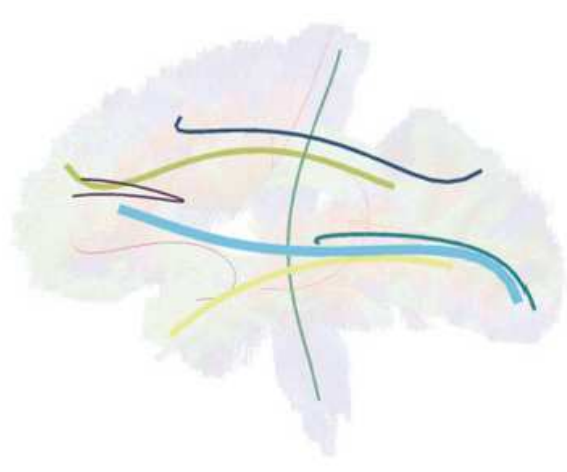

(d) Spatial distribution of WM volume differences

\section{Figure 3}

Whole brain and tract volume fractions in TD and 22q11.2DS participants. (a) Total brain volume and gray matter (GM), white matter (WM), and cerebrospinal fluid (CSF) volume fractions are correlated with age. Points are highlighted for ASM (filled circles) and 22q11.2DS participants (filled squares). The black line denotes the linear regression line for the TD group only (with dashed $95 \%$ confidence intervals). (b) Nominal differences in GM, WM, and CSF are shown for volume fraction estimates from the linear regression for youngest (8 year old) and oldest (18 year old) TD participants. In addition, measured volume fractions are shown for ASM and 22q11.2DS participants (data points overlaid on bar). (c) Individual WM tract volume fractions are shown for (top) youngest (8 year old) and oldest (18 year old) TD, and (bottom) ASM and 22q11.2DS participants. (d) Percent differences in WM tract volume fractions for ASM and 22q11.2DS participants are overlaid on a single subject's tractogram. The radius of each tract 'tube' reflects the percent difference between groups (range from 0-35\%). Tract colors match panel C.

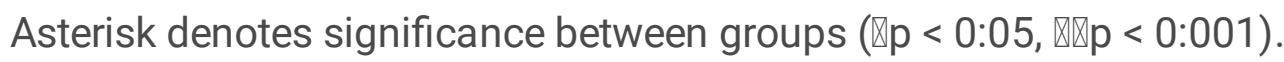




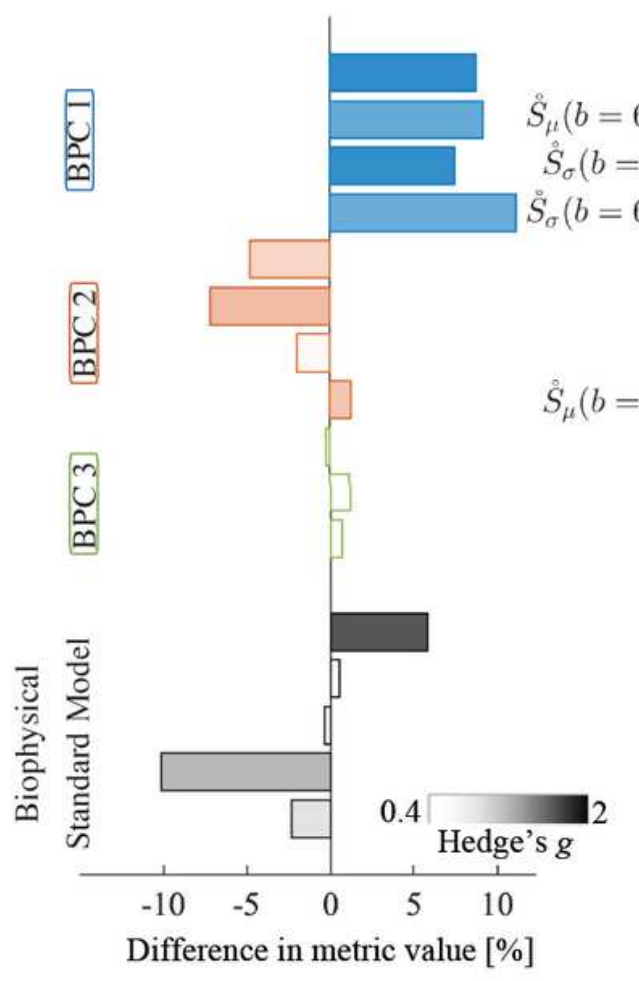

(a) Effect size: total white matter

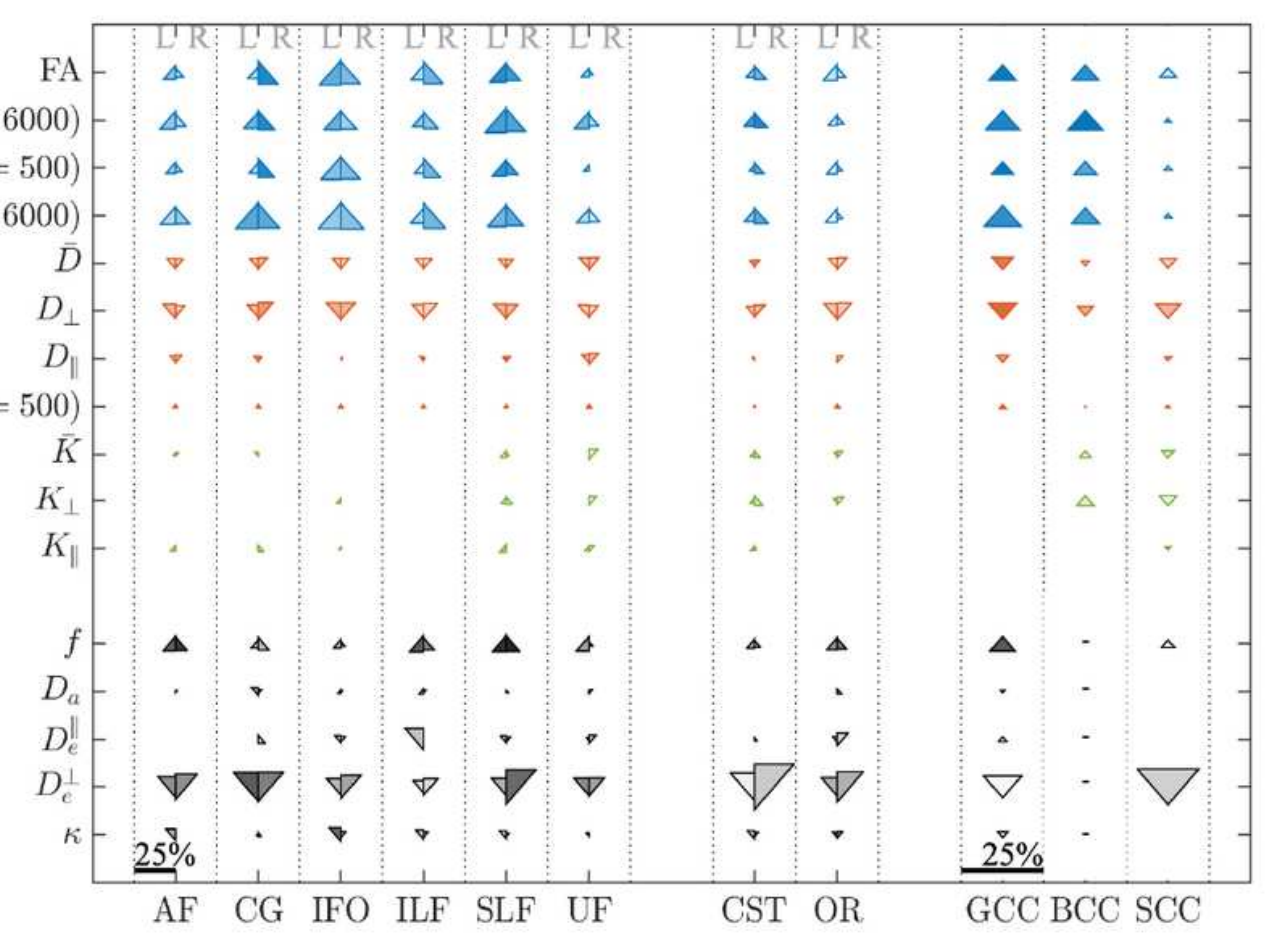

(b) Effect size: individual white matter tracts

\section{Figure 4}

dMRI fingerprint of 22q11.2DS. Metrics are grouped by BPC1 (blue), BPC2 (red), BPC3 (green), and Biophysical Standard Model parameters (black). (a) Bar plots are encoded by mean difference and effect size in total white matter. Color intensity is effect size (Hedge's g); bar length is percent difference between average metric values for ASM and 22q11.2DS participants. (b) Triangle plots are the same statistics for individual white matter tracts. Triangles are divided by left $(L)$ and right $(R)$ hemisphere for association and projection tracts. Commissural tracts span both hemispheres and are not divided. Percent differences are denoted by the scale bar, reaching a maximum of $25 \%$ difference. Triangles pointed up indicate a higher metric values for 22q11.2DS relative to ASM (and vice versa for down). Data from BCC is not shown for the Biophysical Standard Model parameters due to lack of robustness in the along-tract profiling for that particular tract (marked as -). Triangles are omitted for low effect sizes, Hedge's $\mathrm{g}<0: 2$, and blank for Hedge's $\mathrm{g}<0: 4$. 